\title{
Aceleradores para Colombia
}

\author{
Bernardo Gómez Moreno
}

Profesor Emérito, Grupo de Física de Altas Energías, Departamento de Física, Universidad de los Andes, Bogotá, Colombia

Gómez Moreno, B.: Aceleradores de Partículas para Colombia. Rev. Acad. Colomb. Cienc. 38 (Supl.): 71-88, 2014. ISSN 0370-3908.

\section{Resumen}

Se presentan los fundamentos y desarrollo histórico de los aceleradores de partículas, detallando en el sincrotrón, en el principio de estabilidad de fase, principio de sincrotrón. Se introducen los anillos de almacenamiento, acumuladores como sincrotrones de campo magnético constante. Para sincrotrones de electrones, se presenta la radiación de sincrotrón y el aprovechamiento de esta radiación con los onduladores como fuentes de rayos-X. Se destacan las propiedades de la radiación de onduladores para aplicaciones en ciencias, tecnologías e industria. Esto motiva para presentar una propuesta de un centro de radiación de sincrotrón para Colombia, con sincrotrón de electrones en modo de anillo de almacenamiento y onduladores como emisores de rayos-X, con múltiples aplicaciones para investigación, desarrollo e innovación en ciencia, tecnología e industria.

Palabras clave: Aceleradores de partículas, sincrotrones, radiación de sincrotrón, onduladores.

Particle accelerators for colombia

\begin{abstract}
The foundations and historical development of particle accelerators are presented in this article, detailing on the synchrotron, the principle of phase stability, synchrotron principle. Storage rings, synchrotrons at constant magnetic field, are introduced. For the electron synchrotron, synchrotron radiation is presented and how to exploit it with the use of undulators as X-ray sources. Radiation properties of undulators are highlighted for applications in science and industry. This motivates to present a proposal for a synchrotron radiation center for Colombia with an electron synchrotron operating as storage ring and undulators as X-ray emitters, for multiple applications for research, development and innovation in science, technology and industry.
\end{abstract}

Key words: Particle accelerators, synchrotrons, synchrotron radiation, undulators.

\section{Introducción}

En el mundo de los aceleradores de partículas de hoy existen aproximadamente unos 15000 aceleradores. De ellos la gran mayoría son instalaciones comerciales que se encuentran en hospitales y están dedicadas exclusivamente a la medicina nuclear, para diagnóstico, terapia y para la producción de isótopos radioactivos de múltiples aplicaciones en la medicina. Hay también aceleradores de aplicación en la industria en los países desarrollados y, claro está, también los institutos de investigación y laboratorios en las diversas ciencias naturales cuentan con sus propios aceleradores, principalmente en la física nuclear y en la física de altas energías, donde se destacan las mayores instalaciones del mundo como el Laboratorio CERN ${ }^{1}$ en Ginebra, Suiza.

\footnotetext{
${ }^{1}$ CERN: Organización Europea para la Investigación Nuclear, Ginebra, Suiza. http://home.web.cern.ch/
}

En ese mundo de los aceleradores de partículas Latinoamérica cuenta con instalaciones de aceleradores en los hospitales para la medicina, principalmente con aceleradores lineales de electrones que generan rayos de fotones para radioterapia. Pero en la investigación Latinoamérica está muy modestamente representada en el mundo de los aceleradores, apenas con el Laboratorio de Campinas en Brasil, con la "Fuente de Luz Sincrotrón" " y en Argentina, en Buenos Aires con el "Tandar", (instalación mediana de Tandem Van de Graaff actualizado a Pelletron).

Con excepción de Brasil, Latinoamérica está en mora de entrar en los desarrollos más modernos del mundo de los aceleradores de partículas.

\footnotetext{
${ }^{2}$ LNLS: Laboratorio Nacional de Luz Sincrotrón, Campinas, SP, Brasil. http://nls.cnpem.br/

3 TANDAR: Laboratorio TANDAR, Buenos Aires, Argentina. http:/www.tandar.cnea.gov.ar/
} 


\section{Aceleradores de Partículas}

Aceleradores de partículas son máquinas que aceleran partículas cargadas eléctricamente mediante campos eléctricos para aumentar su energía, lo que se manifiesta inicialmente a energías no relativistas como un aumento de la rapidez de las partículas (aceleración) y luego acercándose a la velocidad de la luz, en el rango relativista, como un aumento de la masa $\gamma m_{0}$ de acuerdo con la relación

$$
E=m c^{2}=\gamma m_{0} c^{2}
$$

donde $m_{0}$ es la masa en reposo de la partícula y

$$
\gamma=\frac{1}{\sqrt{1-\beta^{2}}} \quad, \quad \beta=\frac{v}{c},
$$

siendo $v$ la velocidad de la partícula.

La energía que reciben las partículas provienen de campos eléctricos. Se utilizan también campos magnéticos, pero no para aumentar la energía de las partículas, sino para guiarlas en trayectorias curvadas, incluso cerradas en aceleradores en anillo, y para enfocar los rayos de partículas mediante lentes magnéticas, cuadrupolos magnéticos principalmente. Dado que la fuerza magnética es perpendicular a la velocidad, el campo magnético puede cambiar la dirección de movimiento de la partícula pero no puede enriquecer energéticamente a la partícula: La potencia suministrada del campo magnético a la partícula es siempre cero: $\vec{F}_{\text {magn }} \cdot \vec{v}=0$.

Se aceleran principalmente protones(+) y electrones(-) y su antimateria, sus antipartículas, antiprotones(-) y positrones(+). Todas estas son partículas estables. Hasta el presente todos los aceleradores en funcionamiento en el mundo se restringen solo a acelerar partículas estables. Excepcionalmente para el caso de muones, inestables que con vida media de 2.2 microsegundos decaen en electrones y antineutrinos, está en desarrollo un primer acelerador ${ }^{4}$, motivado por la necesidad de superar el efecto de radiación de sincrotrón en aceleradores de electrones en anillo, que limita la energía alcanzable por pérdidas por radiación, efecto despreciable para muones por su mayor masa, 200 veces mayor que la de electrones.

Se aceleran también iones pesados, esto es núcleos atómicos más allá del hidrógeno simple, desde el deuterio (hidrógeno pesado) hasta el plomo, tanto para estudios de la estructura fundamental de la materia, por ejemplo colisiones plomo

\footnotetext{
${ }^{4}$ MAP: Muon Accelerator Program, Fermilab, Batavia, Illinois, Estados Unidos. http://map.fnal.gov/
}

\section{Correspondencia:}

Bernardo Gómez Moreno,bgomez@uniandes.edu.co

Recibido: 18 de junio de 2014

Aceptado: 17 de octubre de 2014 contra plomo para el estudio del plasma de quarks y gluones ${ }^{5}$, como para aplicaciones en la medicina, por ejemplo terapia hadrónica ${ }^{6}$ contra el cáncer con rayos de ${ }^{12} C$.

Siendo los protones notoriamente más pesados que los electrones

$$
m_{\text {protón }} \approx 1830 \cdot m_{\text {electrón }},
$$

se diferencian los aceleradores de protones notoriamente de los aceleradores de electrones, por presentar los electrones muy rápidamente efectos relativistas, como el aumento relativista de la masa, la igualdad (aproximada) de velocidades muy cercanas a la velocidad de la luz, la radiación de sincrotrón, entre otros. Pero también desde el punto de vista de la estructura de la materia hay diferencias notables, por ser los electrones partículas elementales sin estructura interna hasta donde se conoce a las escalas de energía alcanzables en la actualidad, mientras que los protones tienen estructura interna, están constituidos por quarks intercambiando gluones, son así sistemas complejos de partículas elementales que participan de la interacción fuerte que es ignorada por los electrones. Las partículas aceleradas utilizadas como proyectiles para incidir sobre la materia a estudiar, llevan a procesos diferentes según las interacciones fundamentales de las que participan. También que los proyectiles sean elementales o no, hace diferencia importante a las más altas energías, porque para electrones, siendo elementales, la totalidad de la energía que llevan cuenta para el proceso de colisión, mientras que en el caso de protones interaccionan sus "partones", que llevan solo una parte de la energía total del protón, así que solo una fracción de su energía está disponible para el proceso.

Rayos de partículas provenientes de los aceleradores se utilizan para hacer visible la estructura de la materia a escala atómica y subatómica, como radiación que incide sobre el objeto a observar, donde la longitud de onda de la radiación incidente determina la resolución espacial alcanzable, el tamaño de lo más pequeño que se puede distinguir. Con aceleradores de partículas se logran longitudes de onda más y más pequeñas, entre mayor sea el momento que alcancen las partículas aceleradas, entre mayor sea su energía. La longitud de onda de 'de Broglie' $\lambda_{B}$ se relaciona con el momento y energía de las partículas aceleradas:

$$
\lambda_{B}=\frac{h}{p}=\frac{h c}{\sqrt{E_{c i n} \cdot\left(E_{\text {cin }}+2 m_{0} c^{2}\right)}},
$$

\footnotetext{
${ }_{5}^{5}$ ALICE: Experimento ALICE con el Gran Colisionador de Hadrones LHC del Laboratorio CERN. http://home.web.cern.ch/ about/experiments/alice

${ }^{6}$ HIT: Heidelberg Ionenstrahl Therapiezentrum, Centro de terapia hadrónica, Universidad de Heidelberg, Heidelberg, Alemania. http://www.hit-heidelberg.com
} 
(3)

$$
\begin{gathered}
h=6.626068 \cdot 10^{-34} \mathrm{JS} \\
h c=197.32696 \mathrm{MeV} \cdot \mathrm{fm}
\end{gathered}
$$

Se logra así pasar de los micrómetros alcanzables con luz visible $(\lambda \approx 500 \mathrm{~nm})$ a longitudes de onda de

$$
\lambda_{B}=1.24 \cdot 10^{-17} \mathrm{~m}
$$

para electrones $\left(m_{0} c^{2}=0.511 \mathrm{MeV}\right)$ con $E_{\text {cin }}=100 \mathrm{GeV}$, como fue en el acelerador colisionador LEP (Large Electron Positron Collider), Gran Colisionador de Electrones con Positrones del Laboratorio CERN, que estuvo en operación entre los años 1989 y 2000, o longitudes de onda más pequeñas,

$$
\lambda_{B}=1.77 \cdot 10^{-19} \mathrm{~m}
$$

para protones $\left(m_{0} c^{2}=0.938 \mathrm{GeV}\right)$ con $E_{\text {cin }}=7000 \mathrm{GeV}$, como las energías alcanzables actualmente con el acelerador colisionador LHC (Large Hadron Collider) Gran Colisionador de Hadrones del Laboratorio CERN, en operación desde el año 2011.

Con rayos de partículas aceleradas con los aceleradores de más altas energías del mundo, que son los ejemplos aquí dados, se observa la materia hasta esa escala de tamaños, lo más pequeño, $10^{-17} \mathrm{~m}$ y $10^{-19} \mathrm{~m}$. Tanto quarks como leptones aparecen a esa escala de tamaños como puntuales sin manifestar directamente estructura interna alguna, puntuales sin

\begin{tabular}{|c|c|c|c|c|}
\hline \multicolumn{5}{|c|}{ Protones: $\mathrm{m}_{0} \mathrm{c}^{2}=0.938 \mathrm{GeV}$} \\
\hline $\begin{array}{l}\text { Energía total } \\
\text { E }\end{array}$ & $\mathrm{pc}$ & $\begin{array}{c}\beta=v / c \\
\beta=p c / E\end{array}$ & $\gamma=\mathrm{E} / \mathrm{m}_{0} \mathrm{c}^{2}$ & $\lambda$ Broglie $\times 10^{19}$ \\
\hline$[\mathrm{GeV}]$ & {$[\mathrm{GeV}]$} & adimensional & adimensional & {$[\mathrm{m}]$} \\
\hline 1 & 0,35 & 0,3466 & 1,07 & 35766,70 \\
\hline 10 & 9,96 & 0,9956 & 10,66 & 1245,29 \\
\hline 100 & 100,00 & 1,0000 & 106,61 & 123,99 \\
\hline 1000 & 1000,00 & 1,0000 & 1066,10 & 12,40 \\
\hline 10000 & 10000,00 & 1,0000 & 10660,98 & 1,24 \\
\hline \multicolumn{5}{|c|}{ Protones: $\mathrm{m}_{0} \mathrm{c}^{2}=0.000511 \mathrm{GeV}$} \\
\hline $\begin{array}{l}\text { Energía total } \\
\text { E }\end{array}$ & $\mathrm{pc}$ & $\begin{array}{c}\beta=v / c \\
\beta=p c / E\end{array}$ & $\gamma=\mathrm{E} / \mathrm{m}_{0} \mathrm{c}^{2}$ & $\lambda$ Broglie $\times 10^{19}$ \\
\hline$[\mathrm{GeV}]$ & {$[\mathrm{GeV}]$} & adimensional & adimensional & {$[\mathrm{m}]$} \\
\hline 0,1 & 0,10 & 0,999987 & 195,69 & 123981,62 \\
\hline 1 & 1,00 & 1,000000 & 1956,95 & 12398,00 \\
\hline 10 & 10,00 & 1,000000 & 19569,47 & 1239,80 \\
\hline 100 & 100,00 & 1,000000 & 195694,72 & 123,98 \\
\hline 1000 & 1000,00 & 1,000000 & 1956947,16 & 12,40 \\
\hline \multicolumn{5}{|c|}{ Se ha utilizado en esta tabla: } \\
\hline \multicolumn{3}{|c|}{$p c=\sqrt{E^{2}-\left(m_{0} c^{2}\right)^{2}}$} & \multicolumn{2}{|c|}{$\beta=\frac{v}{c}=\frac{m c \cdot v}{m c \cdot c}=\frac{p c}{E}$} \\
\hline \multicolumn{3}{|c|}{$h c=1.2398 \cdot 10^{-15} \mathrm{GeV} \cdot \mathrm{m}$} & \multicolumn{2}{|c|}{$\lambda$ Broglie $=\frac{h}{p}=\frac{h c}{p c}$} \\
\hline
\end{tabular}
volumen observado, son partículas elementales.

Tabla 1. Cantidades relativistas en función de la energía cinética para protones y electrones.
La Tabla 1 presenta para protones y electrones por separado las cantidades relativistas $\beta$ y $\gamma$ en función de la energía total de la partícula, así como su longitud de onda de 'de Broglie'. Los datos presentados resultan de aplicar la relación de 'de Broglie' $p=h / \lambda$ y la expresión relativista momento energía:

$$
E=\sqrt{m_{0}^{2} c^{4}+p^{2} c^{2}} \quad \gamma=E / m_{0} c^{2}
$$

\section{Estructura de Instalación de Acelerador}

Aceleradores de partículas son siempre parte de una instalación, donde la máquina de acelerar es central, requiere una entrada y suministra una salida: Se tiene una fuente de partículas a acelerar, una fuente de iones, donde sistemas neutros de partículas (moléculas, átomos) son ionizados y estos iones siempre cargados eléctricamente son conducidos por un sistema de transporte de haz hasta el acelerador, la máquina donde campos eléctricos entregan energía a las partículas, y éstas ahora más energéticas pasan al sistema de extracción del haz y sistema de transporte, que las llevan del acelerador al blanco (objeto a estudiar), donde inciden y son dispersadas, o generan nuevas partículas, que son registradas por los detectores de partículas. Ver Figura 1.

Es así como se aprende sobre el objeto de estudio (molécula, átomo, núcleo atómico, nucleón), registrando y analizando la radiación que suministró el acelerador e incidió en el objeto, donde fue dispersada y finalmente dejó huella en el detector. Ha sido así en la física atómica y molecular, en la física nuclear, incluso en la física de partículas elementales y esta modalidad de exploración de la materia se denomina "experimentos de blanco fijo". También la mayoría de aplicaciones de aceleradores fuera de la física de altas energías sigue esta modalidad.

Pero en los últimos 30 años, la física de más altas energías ha desarrollado un tipo de experimentos muy sofisticado y exigente, donde el objeto sobre el que incide rayo de

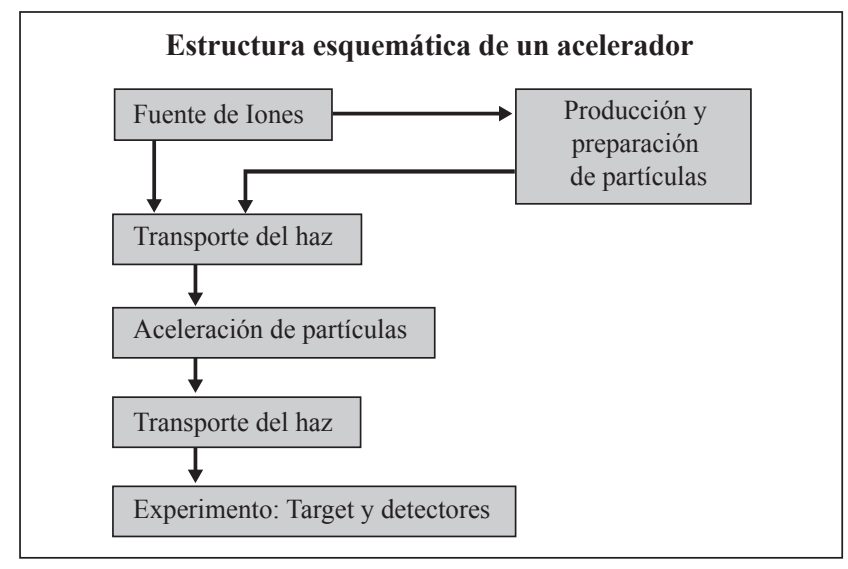

Figura 1. Estructura esquemática de un acelerador 
partículas del acelerador no es un blanco fijo, sino otro rayo de partículas que viene en dirección opuesta. Se tienen así colisiones frontales de partículas que en el punto de colisión reúnen muy alta energía, que se materializa acorde con la equivalencia de masa y energía, $E=m c^{2}$, para producir nuevas partículas según las leyes fundamentales de la naturaleza, surgiendo materia y antimateria, partículas y antipartículas, como en los primeros instantes del Universo luego del Big Bang que le dio origen. Los detectores registran las nuevas partículas, incluyendo el patrón completo de productos de la colisión que constituyen un evento y contiene información sobre las partículas y sus interacciones. Estos son "experimentos de colisionadores", como los realizados por cerca de 30 años con el colisionador protón-antiprotón Tevatrón del Laboratorio Fermilab ${ }^{7}$ en Batavia, Illinois, Estados Unidos, o actualmente con el Gran Colisionador de Hadrones LHC del Laboratorio CERN en Ginebra, Suiza.

\section{Diversos Tipos de Aceleradores de Partículas}

Además de reconocer como máquinas diferentes con sus características propias a los aceleradores de protones (aceleradores hadrónicos) por un lado, y a los aceleradores de electrones (aceleradores leptónicos) por otro lado, también se clasifican los aceleradores según el principio de aceleración de las partículas. Distinguimos los siguientes tipos de aceleradores [Se07], [Wi05], [Wi06], [Co08]:

Aceleradores electrostáticos, que aceleran las partículas con campos eléctricos constantes, que no varían con el tiempo.

Aceleradores por resonancia, donde un generador externo suministra un campo eléctrico oscilante, cuya frecuencia coincide, es resonante, con la frecuencia del movimiento repetitivo de las partículas dentro de la máquina, al pasar en una secuencia lineal de una unidad a otra, donde encuentra los campos eléctricos (por ejemplo el acelerador lineal), o al repetir millones de veces el paso por la misma unidad de aceleración siguiendo trayectorias circulares, o en espiral, guiadas por un campo magnético (por ejemplo la trayectoria en espiral en el ciclotrón).

Aceleradores por inducción, donde las partículas son aceleradas por un campo eléctrico inducido por la variación temporal de un campo magnético (por ejemplo el betatrón).

Aceleradores por principio de sincrotrón, principio de estabilidad de fase, donde un aumento del campo magnético consigue que las partículas atraviesen la unidad de aceleración en el instante justo cuando el campo eléctrico tiene la magnitud requerida para aumentar la energía en la cantidad justa para que el radio de la trayectoria circular de las partículas permanezca constante.

\footnotetext{
${ }^{7}$ Fermilab: Fermi National Accelerator Laboratory, Batavia, Illinois, Estados Unidos. http://www.fnal.gov/
}

Los diversos tipos de aceleradores han surgido de la necesidad de alcanzar energías cada vez mayores, por encima del rango de las energías posibles de los aceleradores del momento. Con gran ingenio en la aplicación de los conocimientos sobre electromagnetismo, sobre el movimiento de cargas eléctricas en campos eléctricos y magnéticos, se ha logrado crear nuevos tipos de aceleradores que han permitido llegar hasta energías de $\mathrm{TeV}\left(10^{12} \mathrm{eV}\right)$. Y este desarrollo tecnológico no se detiene.

Se inició en la década de los años 1920 en Karlsruhe, Alemania, con el estudiante de ingeniería eléctrica Rolf Wideröe, noruego [Wa90]. A partir de 1923 Wideröe inició una serie de publicaciones en la revista "Archiv für Elektrotechnik", donde presentó aplicaciones de electromagnetismo para lograr altos voltajes y así grandes campos eléctricos para acelerar partículas. Sus artículos de 1923 y 1924 presentaron las ideas fundamentales de los aceleradores por resonancia, ciclotrón y acelerador lineal [Wi23]. Luego en 1928 publicó cómo obtener con un transformador por inducción electromagnética, ley de Faraday, un campo eléctrico inducido que acelera electrones. Presentó su idea como "transformador de rayo", que es el principio del betatrón [Wi28].

A partir de las ideas de Wideröe se desarrollaron los primeros aceleradores: En 1931 en la Universidad de California, Berkeley, Ernest Orlando Lawrence y David Sloan construyeron y operaron exitosamente el primer acelerador lineal y el primer ciclotrón, ambos aceleradores por resonancia propuestos por Wideröe [S131], [La32], [La36]. En ese mismo año de 1931 en la Universidad de Princeton y luego en MIT Robert Van de Graaff construyó exitosamente el generador electrostático que lleva su nombre, alcanzando 7 millones de voltios [Gr33]. Luego en 1932 en el Laboratorio Cavendish en Cambridge, Inglaterra, los colaboradores del Profesor Ernest Rutherford, John Cockcroft y Ernest Walton, alcanzaron energías de $400 \mathrm{keV}$ con el "multiplicador de voltaje", hoy conocido como acelerador electrostático Cockcroft-Walton [Co32].

Estos aceleradores fueron creciendo en tamaño y en máxima energía alcanzable, tanto para protones, como para electrones [La51]. En el caso del ciclotrón pronto se reconoció el problema de aumento relativista de la masa de los electrones, que es grande en los electrones y rompía la resonancia entre la frecuencia del movimiento en espiral de los electrones y la frecuencia del generador externo que da el campo eléctrico para acelerar las partículas. La máquina exige mantener la resonancia, así que falla para electrones relativistas, mientras que es buen acelerador para protones y para iones pesados no relativistas a las energías de $\mathrm{MeV}$. Este problema motivó el desarrollo de un acelerador diferente para electrones y la solución estuvo en la idea de la aceleración por inducción con el transformador de rayo de Wideröe de 1928. 
En la Universidad de Illinois, Urbana, en 1940 Donald Kerst perfeccionó la idea del transformador de rayo y su diseño, el betatrón, constituyó un muy exitoso invento para acelerar electrones hasta energías de cientos de $\mathrm{MeV}$, que acelerador que se mantuvo vigente hasta finales de la década de 1970 como el acelerador para aplicaciones médicas en terapia del cáncer [Ke40], [Ke41], [Ke42].

Los aceleradores circulares, donde las partículas guidas por un campo magnético pasan repetidamente por la misma unidad de aceleración, se enriquecieron especialmente con el invento del sincrotrón en 1940. Desde entonces este es el acelerador en anillo más exitoso, el acelerador moderno, que en versiones de anillos acumuladores y colisionadores, lleva a las más altas energías logradas en los mayores laboratorios como Fermilab y CERN.

Los diversos tipos de aceleradores, luego de ser superados por nuevos desarrollos tecnológicos requeridos para alcanzar mayores energías que las máximas logradas por los ya existentes, no desaparecen definitivamente. Si el rango de energías de un acelerador es adecuado para cierta área de investigación que no requiere las más altas energías o para aplicaciones en la industria, o en la medicina, el acelerador se mantiene, mientras su operación sea razonable hasta ser superado en los demás aspectos tecnológicos, o de costos de operación, como en el caso ya mencionado del betatrón. La Figura 2 presenta el "diagrama de Livingston" que muestra los diversos tipos de aceleradores según su rango de energías graficado como línea de tiempo desde 1932 hasta 1965. Se reconoce allí la vigencia de cada tipo de acelerador y se destaca la frontera de las más altas energías, que ha ido aumentando continuamente desde la invención de los primeros aceleradores [Li62].

\section{El Sincrotrón}

A comienzos de la década de 1940 Edwin McMillan en la Universidad de Berkeley, California, y Vladimir Veksler en la Unión Soviética, llegaron independientemente, y sin conocimiento el uno del otro, a la idea de la "estabilidad de fase", también llamada "principio de sincrotrón", que es la base del funcionamiento del acelerador en anillo de mayor importancia en el mundo y que luego de 60 años sigue siendo el acelerador moderno, el más versátil y a la vez el más poderoso, el sincrotrón [Mc45], [Ve44], [Bo46]. Ver Figura 3.

En el sincrotrón, las partículas a acelerar (protones, antiprotones, iones pesados, o también electrones, o positrones) siguen una trayectoria cerrada, en anillo, en el diseño original una trayectoria circular de radio $R$. Un campo magnético vertical $B$, perpendicular al plano de la trayectoria actúa a lo largo de todo el recorrido de las partículas dentro de un tubo toroidal que mantiene un alto vacío $\left(10^{-8}\right.$ Torr). El campo

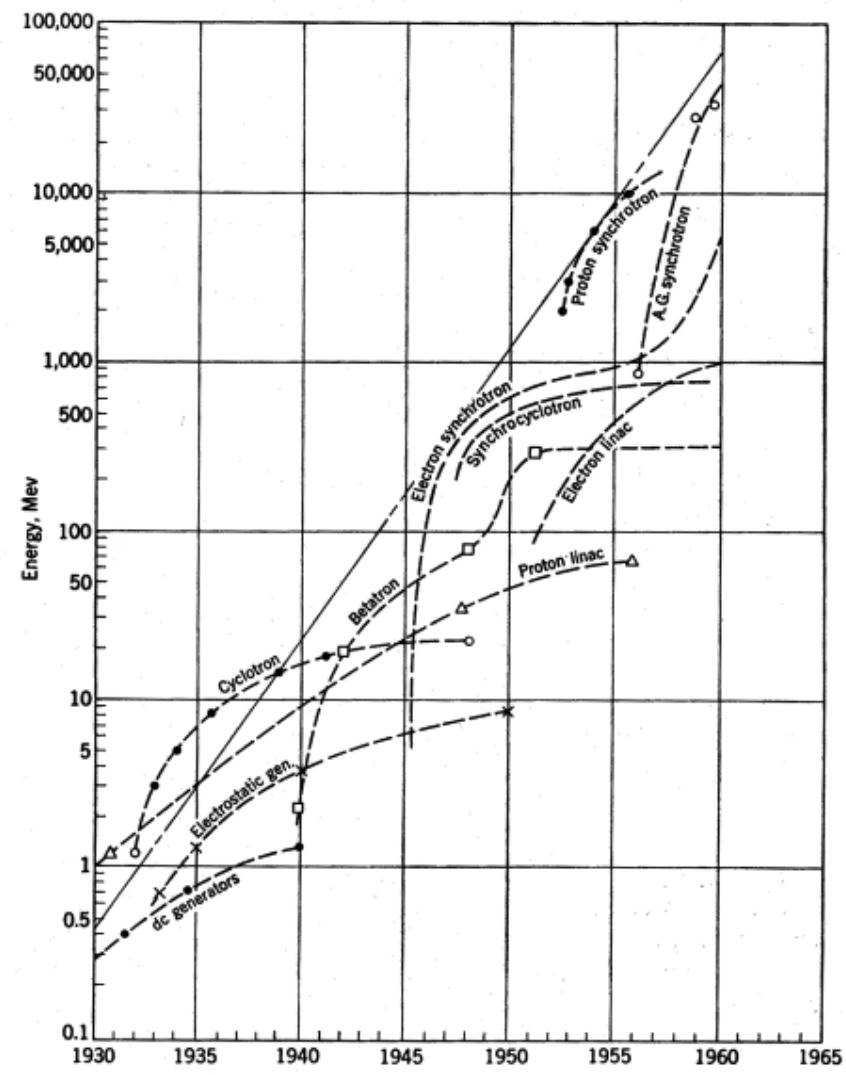

Figura 2. "Diagrama de Livingston": Línea de tiempo de los diversos tipos de aceleradores según su rango de energías. se destaca la frontera de las más altas energías. Fuente: [Li62]

magnético $B$ provee la fuerza centrípeta requerida para el movimiento en circunferencia de las partículas de carga eléctrica $q$, así que la ecuación de movimiento radial es:

$$
\left|\vec{F}_{\text {mag }}\right|=q v B=m \frac{v^{2}}{R}
$$

de donde se obtiene para el radio $\mathrm{R}$ de la trayectoria:

$$
R=\frac{m v}{q B}=\frac{p}{q B}=\text { const. }
$$

En una cavidad de radiofrecuencia $R F$ se tiene el campo eléctrico sinusoide que oscila con una alta frecuencia acorde con el movimiento de las partículas en el anillo, así que estas encuentran siempre el campo eléctrico $E$ con la fase adecuada para ser aceleradas.

El sincrotrón es un acelerador de altas energías, para partículas relativistas, así que es buena aproximación asumir que todas las partículas tienen la misma rapidez, muy cercana a $c=3 \cdot 10^{8} \frac{\mathrm{m}}{\mathrm{s}}$, pero no todas tienen la misma energía, lo que se manifiesta en la masa relativista. Partículas con menor energía, menor momento $\mathrm{p}$, siguen en el campo magnético $\mathrm{B}$ 
una trayectoria circular de menor radio $\left(\mathrm{R}_{<}\right)$que partículas de mayor energía $\left(\mathrm{R}_{-}\right)$. Comparando con la partícula ideal que cumple la relación $R=m v / q B$, esto es que tiene la energía que corresponde al radio $R$ y al campo $B$ de la máquina, partículas de menor energía tienen trayectoria menor y a igual rapidez llegan a la cavidad de radiofrecuencia antes que la partícula ideal, y aquellas de mayor energía, mayor recorrido a igual rapidez, llegan más tarde. Ver Figura 4.

Es así que el campo eléctrico que encuentran las partículas en la cavidad de radiofrecuencia depende de su instante de llegada, y para una fase inicial adecuada del campo eléctrico oscilante se logra que partículas de menor energía encuentren un campo eléctrico mayor para ser más aceleradas, y lo opuesto para partículas de mayor energía, que reciben menor

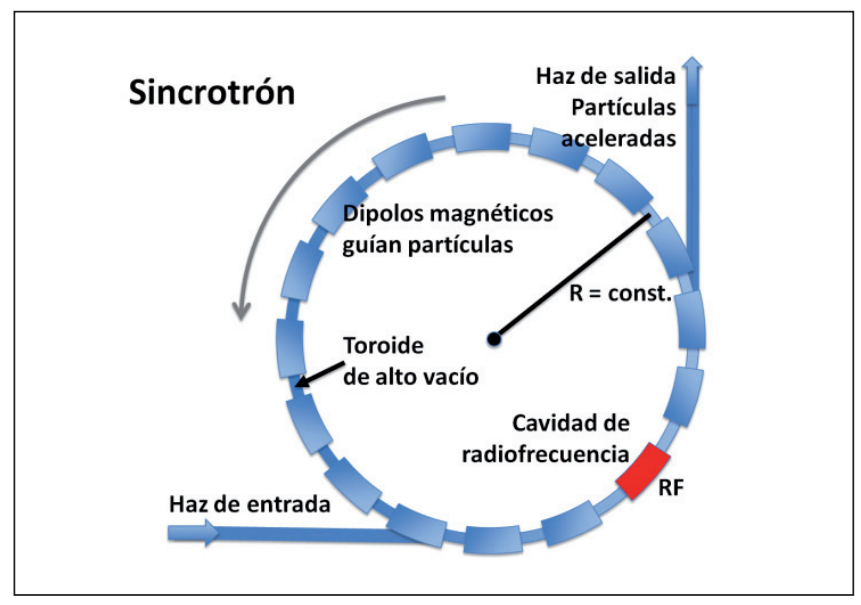

Figura 3. Configuración de un sincrotrón, acelerador en anillo de radio constante, basado en el principio de estabilidad de fase.

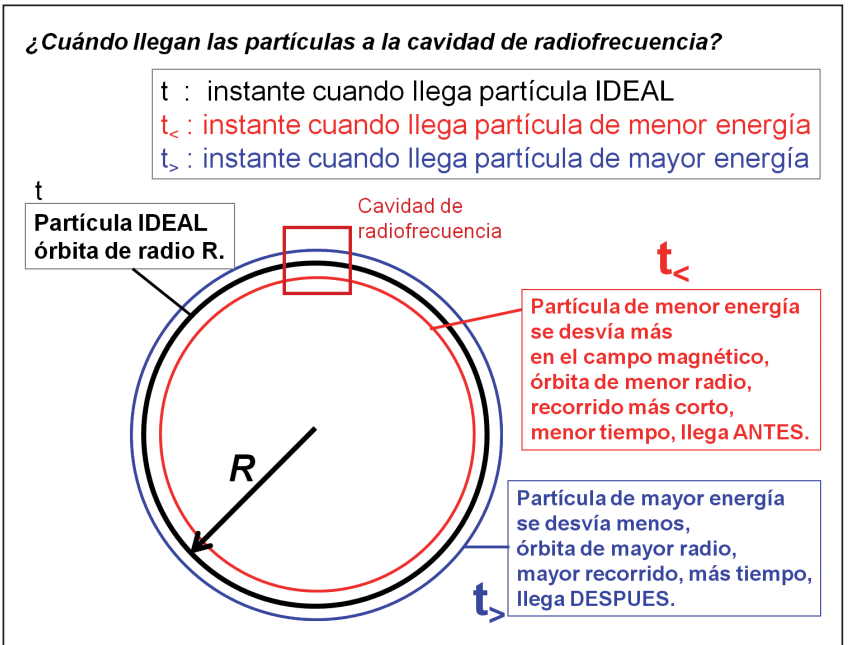

Figura 4. Tiempos de recorrido de una vuelta en el sincrotrón para partículas de diversa energía.
¿Cuándo llegan las partículas a la cavidad de radiofrecuencia?

$\mathrm{t}$ : instante cuando llega partícula IDEAL

$\mathrm{t}_{<}$: instante cuando llega partícula de menor energía

$t_{>}$: instante cuando llega partícula de mayor energía

Campo eléctrico en la cavidad de radiofrecencia:

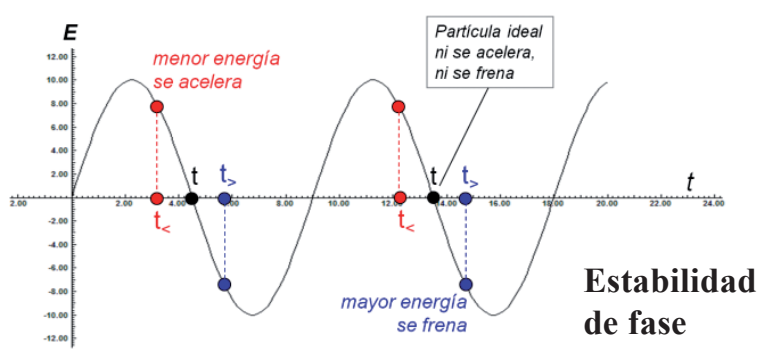

Figura 5. Ilustra la estabilidad de fase o principio de sincrotrón. Se presenta estabilidad para "punto de trabajo" en el flanco descendente de la oscilación sinusoidal del campo eléctrico en la cavidad de radiofrecuencia.

energía para que todas se acerquen a la misma energía de la partícula ideal y con ello se acerquen al mismo tamaño de la trayectoria y a iguales tiempos de recorrido. Se tiene así estabilidad temporal, estabilidad de fase, si la fase inicial del campo eléctrico oscilante se escoge así que la partícula ideal encuentre el campo en el flanco descendente de la oscilación sinusoide como lo muestra la Figura 5.

Si se aumenta el campo magnético $B$ que guía las partículas en el tubo toroidal del sincrotrón, disminuye el radio de la trayectoria circular de todas las partículas así que todas tendrán menor recorrido, llegarán antes de la ideal, y serán aceleradas justo lo requerido para aumentar el momento $p$ así que $p / B$ recupere el valor ideal de diseño de la máquina y el radio $R$ ideal se mantenga constante pero ahora para un campo magnético mayor $(R=p / q B)$. Las partículas circulan ahora con mayor momento $\mathrm{p}$, mayor energía. Se han acelerado respondiendo al aumento del campo magnético $B$. Esto es precisamente lo que caracteriza el sincrotrón: Al aumentar el campo magnético $B$ se aceleran las partículas en la cavidad de radiofrecuencia justo lo requerido para mantener el radio de la trayectoria constante, y esto se logra por la estabilidad de fase, principio de sincrotrón.

Si el campo magnético $B$ de sincrotrón varía como función sinusoide, solo pueden acelerarse partículas mientras el campo $B$ va en aumento, solo durante el intervalo de tiempo que corresponde al flanco ascendente de la función seno, así que se inyectan partículas a la máquina justo después del instante del mínimo de la función seno, se aceleran en millones de revoluciones mientras $B$ va en aumento, y poco antes de alcanzar el máximo de la función seno deben extraerse las partículas de la máquina, como lo muestra la Figura 6. 


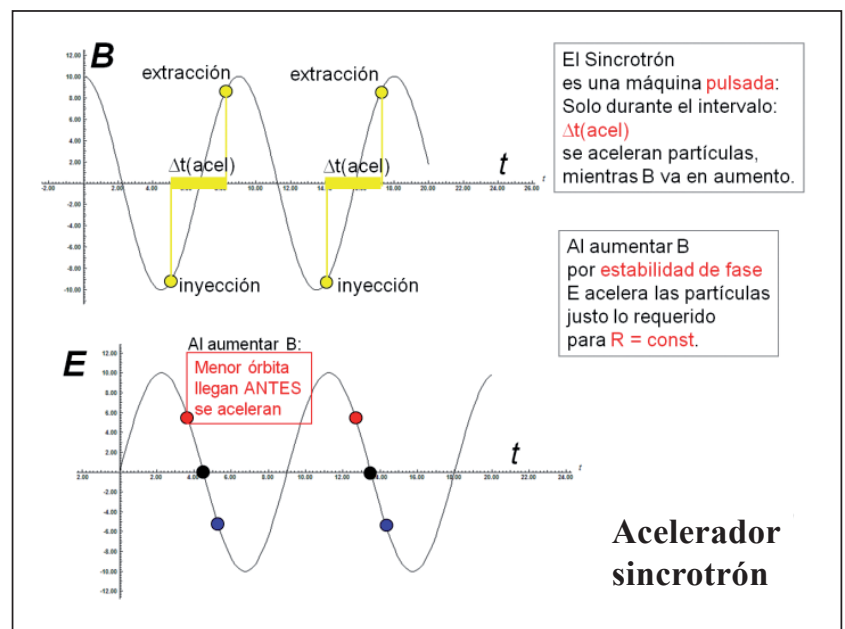

Figura 6. Ilustra por qué el sincrotrón es una máquina pulsada. Solo se aceleran las partículas durante los intervalos de tiempo cuando el campo magnético va en aumento.

El sincrotrón es así una máquina pulsada: Va entregando paquetes de partículas aceleradas, que salen de la máquina a intervalos de tiempo dados por la frecuencia de oscilación del campo magnético $B$.

\section{Anillos de Almacenamiento y Colisionadores}

Es posible mantener a las partículas circulando por muy largo tiempo en el tubo toroidal del sincrotrón, realizando millones de millones de recorridos completos, si el punto de trabajo del campo eléctrico se escoge en el flanco descendente con valor cero para la partícula ideal, que no será acelerada. Para campo magnético $B$ constante, se mantienen así las condiciones de estabilidad en la máquina con la misma energía. La partícula ideal no es acelerada y pérdidas de energía por radiación, como radiación de sincrotrón, se compensan con la aceleración que sí reciben partículas de menor energía que la ideal. La máquina se mantiene en estas condiciones de estabilidad con energía constante y se le llama entonces "anillo de almacenamiento" (storage ring), que son sincrotrones con campo magnético $B$ constante.

Es posible utilizar el anillo del sincrotrón inicialmente como acelerador y luego como anillo de almacenamiento, como se ilustra con la Figura 7. Las partículas provenientes de un preacelerador son inyectadas al anillo, que inicialmente tiene ya el campo magnético adecuado para cumplir con la condición $(R=p / q B)$. Luego el campo magnético va en aumento tipo rampa, hasta alcanzar la magnitud final, que corresponde a la energía final, que es la energía del anillo de almacenamiento. A partir de ese instante se mantiene $B=$ constante, así que el anillo opera como anillo de almacenamiento. Este es el procedimiento que se sigue actualmente con el Gran Colisionador de Hadrones LHC del Laboratorio CERN.

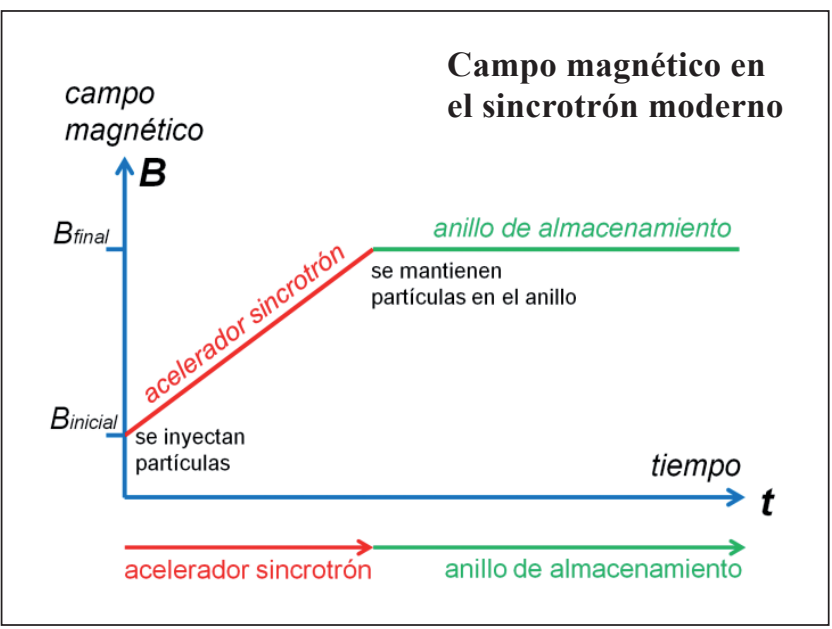

Figura 7. Evolución temporal del campo magnético de un anillo que inicialmente opera como sincrotrón y continúa luego a energía fija como anillo de almacenamiento.

En el sincrotrón y en el anillo de almacenamiento circula un gran número de partículas, reunidas por "paquetes de partículas", llamados "bunches". Hoy se logra reunir hasta $10^{12}$ partículas en un bunch, con alta densidad, así que el bunch de forma cilíndrica tiene un diámetro de apenas micrómetros y una longitud de algunos milímetros.

Los anillos de almacenamiento se emplean hoy para acumular antimateria, recibiendo antipartículas a medida que van siendo generadas por colisiones de proyectiles con suficiente energía, incidiendo sobre bloques de materia pesada (alto número de protones $\mathrm{Z}$ en el núcleo atómico). Así por ejemplo protones con energía mayor a $2 \mathrm{GeV}$ son frenados al incidir sobre wolframio, emitiendo radiación de frenado ( "Bremsstrahlung") con suficiente energía para la producción de pares en protón antiprotón. Los antiprotones son desviados mediante campos eléctricos y magnéticos y llevados al anillo de almacenamiento, donde se acumulan hasta que se alcanza un buen número de antipartículas para formar bunches e inyectarlos en aceleradores mayores.

También se emplean los anillos de almacenamiento (sincrotrones de $B$ constante) como colisionadores. La instalación de sincrotrón se mantiene con campo magnético constante y con cavidad de radiofrecuencia activa, para que ésta por estabilidad de fase recupere la energía que las partículas hayan perdido por efectos de interacción con las demás partículas del bunch o por radiación de sincrotrón. En el mismo anillo pueden circular bunches en direcciones opuestas para colisionar frontalmente, si se trata de partículas de carga eléctrica opuesta, como en colisionadores protón-antiprotón, o electrón-positrón. Para colisiones de partículas de igual carga eléctrica se requieren dos anillos independientes, 
iguales, paralelos pero con campos magnéticos en direcciones opuestas, como en el Gran Colisionador de Hadrones LHC del CERN.

De especial importancia por las aplicaciones son los anillos de almacenamiento de electrones de alta energía en el rango de GeV. Estos anillos son las fuentes de radiación de sincrotrón, también llamadas "fuentes de luz sincrotrón".

\section{Estabilidad Transversal y Radial}

Además de la estabilidad temporal, estabilidad de fase, que es el fundamento del sincrotrón, también se requiere estabilidad espacial, tanto en el plano horizontal de la trayectoria circular de las partículas, como en la dirección vertical. Sin estabilidad las partículas se pierden en la máquina, que deja de funcionar. [Wi05], [Wi06], [Co08]

Hay estabilidad cuando hay oscilaciones alrededor de la posición de equilibrio. Hay oscilaciones transversales, cuando una fuerza restauradora vertical $F_{z}$ actúa sobre la partícula que se aleja verticalmente dejando el plano de movimiento, y es una fuerza proporcional a la desviación: $F_{z}=-k_{z} \cdot z$, donde $z=0$ corresponde al plano de la trayectoria. Ver el sistema de coordenadas utilizado en la Figura 8.

Se logra estabilidad transversal, estabilidad vertical, con líneas del campo magnético curvadas, así que el campo magnético principal $B_{z}$ está acompañado de una componente menor horizontal $B_{x}$, que da la fuerza vertical restauradora. Ver Figura 9.

En el plano de movimiento se tiene estabilidad con una fuerza restauradora en dirección radial, $F_{x}=-k_{x} \cdot x$, midiendo $x$ como desviación en dirección radial a partir del radio $R$ de la trayectoria ideal. Aquí tanto $k_{x}$ como $k_{z}$ dependen de la posición $S$ a lo largo de la circunferencia: $k_{x}(s), k_{z}(s)$.

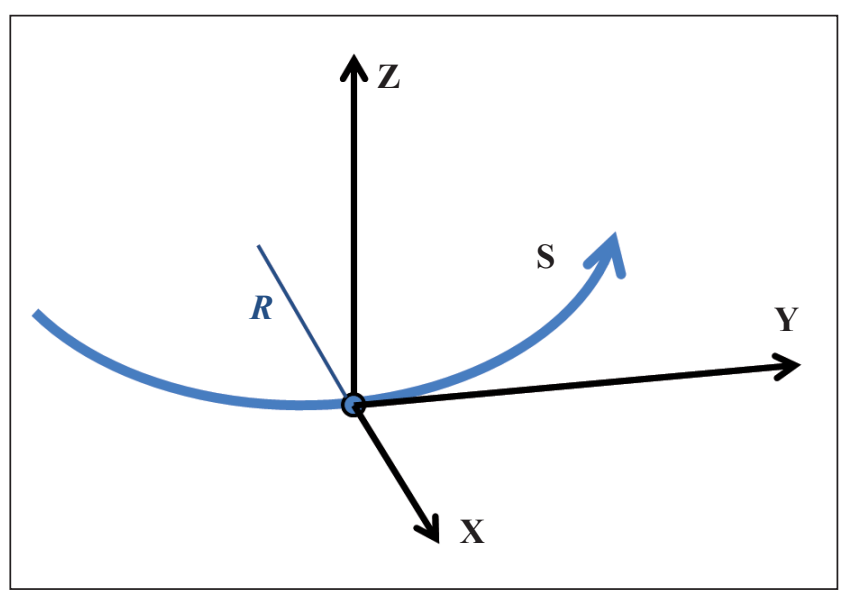

Figura 8. Sistema de coordenadas para descripción del movimiento de las partículas en el sincrotrón.

\section{Estabilidad en la dirección vertical}

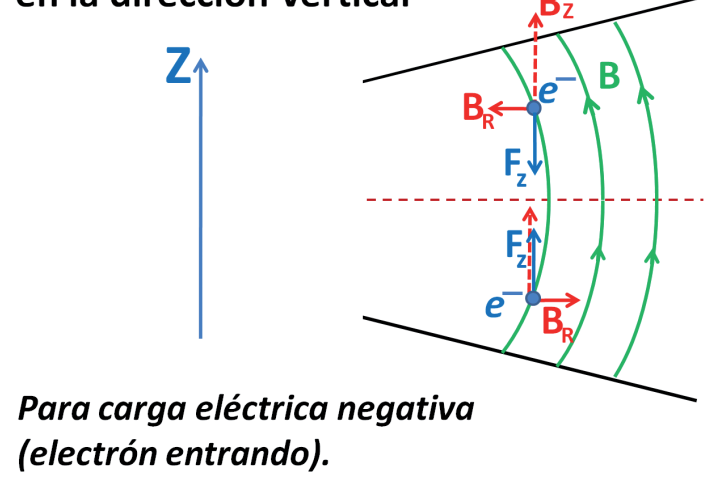

Figura 9. Estabilidad transversal (vertical) mediante líneas de campo magnético curvadas adecuadamente.

Se logra estabilidad radial cuando el campo magnético $B$ varía más lentamente que $1 / R$, como lo ilustra la Fig.10, porque la aceleración centrípeta va como $1 / R$, así que si la partícula se desvía al interior del anillo, $r<R o$, la fuerza magnética resulta menor que lo requerido como fuerza centrípeta, y la partícula tiende a salirse. Si la desviación es hacia fuera del anillo, $r>R o$, la fuerza magnética resulta mayor que lo requerido como fuerza centrípeta y el efecto es hacia el interior. Aquí $R o$ es el radio de la trayectoria de la partícula ideal, que cumple la relación (6): $R_{0}=p / q B$.

Inicialmente en la primera generación de sincrotrones se tenía a lo largo de la circunferencia una secuencia continua de electroimanes dipolares para el campo magnético B del sincrotrón. Este campo además de guiar a las partículas en la trayectoria circular también mantenía la estabilidad transversal con la curvatura de sus líneas de campo y la estabilidad radial disminuyendo más lentamente que $1 / r$. Se lograba así un "enfoque débil" del haz de partículas dentro del toroide del sincrotrón. Pero la amplitud de las oscilaciones alrededor de la trayectoria ideal resultaba muy grande y aumentaba con la energía de las partículas, llegando a oscilaciones de 4 metros de amplitud para protones de 30 $\mathrm{GeV}$, lo que llevaría a un límite del orden de $10 \mathrm{GeV}$ como máxima energía alcanzable para protones.

La solución al problema del enfoque débil se encontró al ensamblar la secuencia de electroimanes bipolares a lo largo del anillo alternando el gradiente del campo magnético B, así que a un electroimán con $\frac{\partial B}{\partial r}<0$, seguía uno con $\frac{\partial B}{\partial r}>0$, aunque este tuviera efecto de desenfoque. Sin embargo la secuencia de electroimanes con gradiente alterno tiene un efecto global de enfoque, se conoce como "enfoque fuerte" y lleva a oscilaciones de amplitud pequeña, muy reducida 


\section{Estabilidad}

en la dirección radial

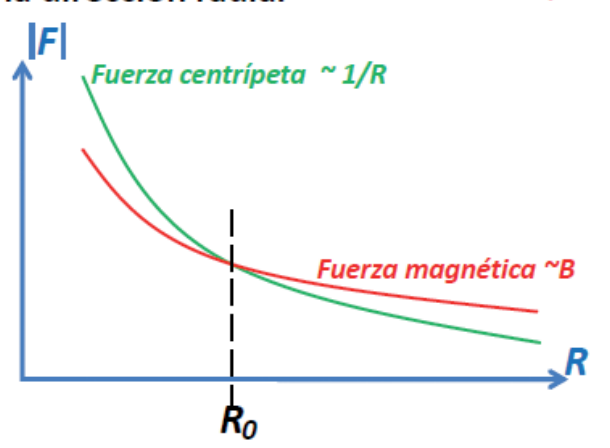

Figura 10. Estabilidad radial en el sincrotrón de enfoque débil: El campo magnético debe disminuir más lentamente que 1/R.

comparada con el "enfoque débil". Así que para protones de $30 \mathrm{GeV}$ las amplitudes de oscilación radial de 4 metros se reducen en un factor de $1 / 20$ a solo $20 \mathrm{~cm}$. Los sincrotrones de "gradiente alterno", "enfoque fuerte", permitieron superar los $50 \mathrm{GeV}$ para protones y constituyen la segunda generación de sincrotrones [Co52], [Co57], [Co58].

La evolución del sincrotrón llevó a una tercera generación al separar la tarea de guiar a las partículas en el anillo mediante dipolos magnéticos de la tarea de enfocar el haz dentro del anillo mediante cuadrupolos magnéticos, manteniendo con los cuadrupolos el esquema de alternar enfoque y desenfoque del "enfoque fuerte". Se organizan así los electroimanes a lo largo del anillo en una secuencia de celdas iguales, cada una compuesta de cuadrupolo de enfoque $(F)$, dipolos de curvar la trayectoria $(O)$, cuadrupolo de desenfoque $(D)$, y se denomina “celda FODO”, que se va repitiendo a lo largo del anillo [Wi06].

La secuencia de celdas FODO da mayor flexibilidad para configurar el sincrotrón, así que la trayectoria de las partículas no tiene que ser necesariamente circular. Hoy los sincrotrones modernos pueden constar de tramos curvados seguidos de tramos rectilíneos donde no se requieren electroimanes dipolares para guiar el haz, reduciendo los componentes del acelerador y con ello reduciendo los costos. Se reemplaza así el anillo circular de la trayectoria de partículas en el sincrotrón por polígonos regulares, configuraciones más sencillas que se adaptan a las necesidades de la instalación del laboratorio [Wi05], [Wi06].

\section{Lentes Magnéticas}

Dentro del sincrotrón moderno, o fuera de él en la línea de conducción del rayo de partículas, se utilizan cuadrupolos magnéticos como lentes magnéticas convergentes $(+)$ o divergentes (-). La Figura 11 representa una lente magnética compuesta por dos cuadrupolos, que se ubican en la línea del haz, uno tras otro. Para partículas de carga eléctrica positiva saliendo del plano de la página el primer cuadrupolo enfoca en la dirección horizontal $x$, desenfoca en la dirección vertical $z$. El enfoque es inverso con el segundo cuadrupolo.

Siempre un solo cuadrupolo magnético enfoca en una dirección y desenfoca en la dirección perpendicular a la primera. Una "lente magnética" consta de dos cuadrupolos seguidos, con igual eje de simetría, donde el segundo cuadrupolo está rotado en 90 grados con respecto al primero. En cada dirección (horizontal y vertical) se tiene enfoque de un cuadrupolo y desenfoque del otro, y los efectos de los dos cuadrupolos se complementan resultando globalmente enfoque en las dos direcciones perpendiculares. Variando la intensidad de la corriente eléctrica en los cuadrupolos, esto es variando la intensidad del campo magnético en cada cuadrupolo, se puede ajustar la distancia focal de la lente magnética.

\section{Radiación de Sincrotrón}

La radiación de sincrotrón es un efecto muy importante que aparece en electrones en movimiento circular o trayectorias curvadas, cuando alcanzan energías relativistas, por ejemplo en sincrotrones, un efecto que aumenta como la cuarta potencia de la energía [Mc46]. Para electrones de $100 \mathrm{GeV}$ acelerados en anillo sincrotrón de $1 \mathrm{~km}$ de radio la pérdida de energía por radiación de sincrotrón no puede compensarse razonablemente en la cavidad de radiofrecuencia, haciendo imposible el funcionamiento del acelerador. Así inicialmente la radiación de sincrotrón aparecía solo como un limitante serio para lograr rayos de electrones de muy altas energías, pero luego al estudiar sus propiedades se reconoció el enorme potencial que tiene como herramienta para aplicaciones en muy diversas áreas de desarrollo tecnológico, innovador. Es una herramienta bastante más poderosa que los rayos-X emitidos por los tubos de Röntgen.

Lente magnética compuesta por dos cuadrupolos
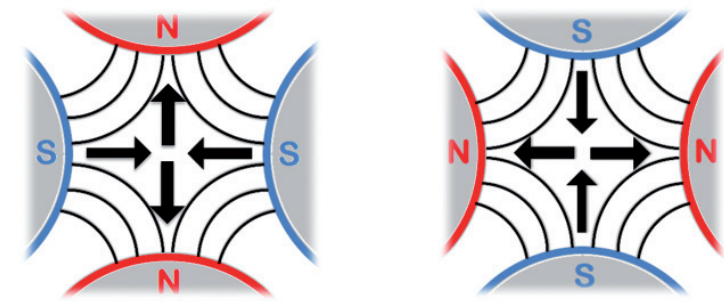

Figura 11: Lente magnética compuesta por dos cuadrupolos, que se ubican uno tras otro. Los dos cuadrupolos alternan polaridad. 
En la década de 1970 se inició muy tímidamente el aprovechamiento de la radiación de sincrotrón emitida tangencialmente al anillo del sincrotrón de electrones de energías de $2 \mathrm{GeV}$ o superiores hasta $10 \mathrm{GeV}$. Se ofreció como servicio a la industria, que alquilaba el tiempo de "luz sincrotrón", generando ingresos para los laboratorios de física de altas energías dedicados hasta ese momento solo a la investigación fundamental. El uso de la radiación de sincrotrón fue así "parásita" de los experimentos de los físicos de altas energías, pero luego en la década de 1980 se establecieron instalaciones mayores, grandes centros de radiación de sincrotrón, dedicados exclusivamente a las aplicaciones de la esta radiación para desarrollos tecnológicos innovadores.

Veamos las propiedades de la radiación de sincrotrón [Ja99], [Wi05]. Toda carga eléctrica acelerada es fuente de radiación electromagnética, que se lleva parte de la energía de la partícula. Así para una partícula de carga eléctrica $q$, que tiene masa en reposo $m_{0}$ y que circula en un anillo de almacenamiento, o acelerador sincrotrón, de radio $R$, la energía radiada en una circunferencia recorrida:

$$
\Delta E_{1 \text { vuelta }}=\text { const } \cdot \frac{1}{\left(m_{0} c^{2}\right)^{4}} \cdot \frac{E^{4}}{R}
$$

como lo presenta Edwin McMillan en "Radiación por un Grupo de Electrones en Movimiento en Órbita Circular", artículo en el Physical Review 68 de 1945, seguido a la presentación de su invención del sincrotrón en la misma revista [Mc45], [Mc46].

Como la energía radiada en cada revolución en el sincrotrón resulta proporcional a $\left(m_{0} c^{2}\right)^{-4}$, el efecto de pérdida de energía por radiación de sincrotrón es dominante en el caso de electrones de masa 1830 veces menor que la de protones:

$$
\frac{P_{\text {rad, electrón }}}{P_{\text {rad, protón }}}=\frac{\left(m_{0 \text { protón }} c^{2}\right)^{4}}{\left(m_{0 \text { electrón }} c^{2}\right)^{4}}=1830^{4} \approx 1.12 \cdot 10^{13}
$$

Así comparando a la misma energía, la radiación de sincrotrón de electrones es $10^{13}$ veces mayor que la de protones. Fuentes de radiación de sincrotrón son por esto sincrotrones de electrones y para aplicaciones resulta óptimo el rango de energía de electrones entre 1 y $10 \mathrm{GeV}$.

Además como la energía radiada en cada circunferencia recorrida aumenta con la cuarta potencia de la energía de la partícula y disminuye con el radio de curvatura, va proporcional a $E^{4} / R$, el efecto es dominante a muy altas energías, por más que se diseñe el anillo del sincrotrón de gran tamaño, $\mathrm{R}$ muy grande. La energía a la cuarta potencia es dominante. Esto es lo que impone el límite a los aceleradores circulares de electrones, pero es a la vez lo que hace atractivo el efecto si se utiliza como fuente de radiación, como "fuente de luz sincrotrón". Anillos pequeños de electrones a altas energías son óptimos como fuente de radiación.
Espacialmente la radiación de sincrotrón aparece emitida tangencialmente a la trayectoria curvada de las partículas, esto es perpendicular a la aceleración centrípeta, en la dirección frontal hacia delante y "colimada" en un cono de ángulo de apertura $\theta=1 / \gamma$, donde $\gamma=E /\left(m_{0} c^{2}\right)$, como lo ilustra la Figura 12. Este cono de radiación es un efecto relativista observado en el marco de referencia del laboratorio. Así para electrones con $m_{0} c^{2}=511 \mathrm{keV}$ y energía total de $10 \mathrm{GeV}$ resulta $\gamma=19570$ así que el ángulo de apertura del cono de radiación es de $\theta=5.1 \cdot 10^{-5}$ radianes, aproximadamente $\theta=0.003^{\circ}$. (Ver referencia [Wi05]).

Aunque el cono de radiación de sincrotrón es altamente colimado con ángulo de apertura tan pequeño, de décimas a centésimas de mili radianes, en el laboratorio se observa el cono desplazándose tangencialmente al electroimán del sector del sincrotrón que abarca el experimento instalado en el exterior del anillo. Y se desplaza a la velocidad de la luz, así que se observa un "abanico continuo de emisión de radiación" que proviene de todo el sector del sincrotrón, como lo muestra la Figura 13. Mediante diafragmas, colimadores diseñados acorde a las necesidades del usuario, se define un "haz de radiación de sincrotrón".

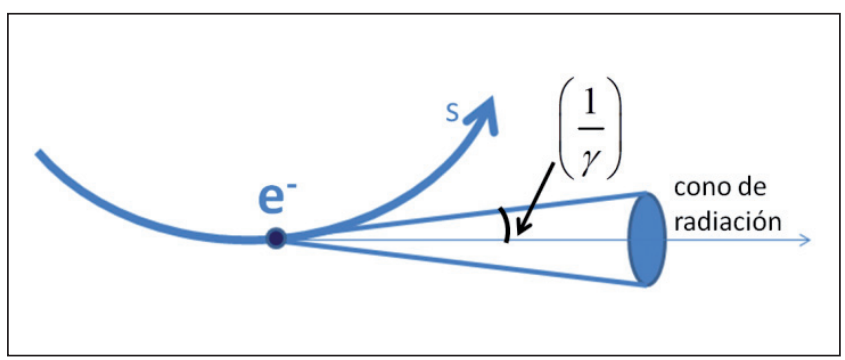

Figura 12: Cono de radiación de sincrotrón

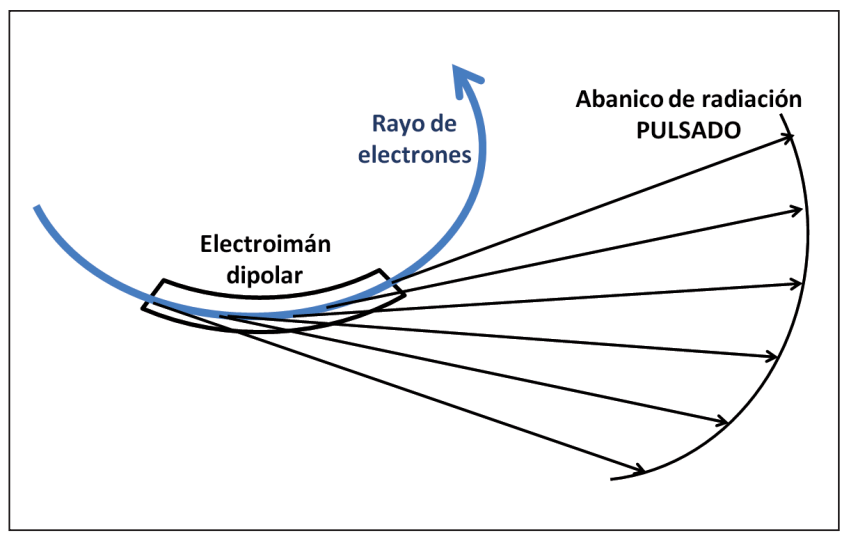

Figura 13: Abanico de radiación de sincrotrón proveniente del haz de electrones que atraviesan el sector del anillo cubierto por el electroimán observado. 
Este haz de radiación de sincrotrón se obtiene pulsado cada vez que un bunch del sincrotrón recorre el sector del acelerador que es observado por el usuario. Así la frecuencia de los pulsos está dada por el producto del número de bunches que circulan en el sincrotrón por la frecuencia de revolución del bunch:

$$
\begin{aligned}
& f_{\text {pulsos }}=N_{\text {bunches }} \cdot f_{\text {rev }}=N_{\text {bunches }} \cdot \frac{1}{(2 \pi R / c)} \\
& f_{\text {pulsos }}=\frac{N_{\text {bunches }} \cdot c}{2 \pi R}
\end{aligned}
$$

\section{Onduladores y Wigglers}

Las ventajas de la radiación de sincrotrón al estar colimada en un cono de ángulo de apertura de solo décimas a centésimas de mili radianes se pierden con el desplazamiento del cono a lo largo del sector observado desde el exterior del anillo del sincrotrón. Recortar el abanico de radiación mediante diafragmas permite definir un haz, una línea de radiación de sincrotrón. Pero puede lograrse algo mejor: Onduladores y wigglers permiten aprovechar significativamente mejor la radiación de sincrotrón [Ja99], [Wi05].

Un ondulador es una unidad compuesta por un arreglo rectilíneo de magnetos que van alternando en polaridad, NS ... SN ... NS ... SN ..., estableciendo un canal de ancho $D$, donde hay campo magnético uniforme, vertical, que periódicamente cambia de dirección, alternando en el sentido vertical. El período de este arreglo se denomina $\lambda_{0} \mathrm{y}$ es constante a lo largo de toda la unidad de varios metros de longitud, $L$, como lo representa la Figura 14. Los magnetos pueden ser electroimanes, o preferiblemente imanes permanentes.

Un rayo horizontal de electrones de energía del orden de 1 $\mathrm{GeV}$ atraviesa el canal del ondulador, encontrando el campo magnético transversal que cambia de dirección periódicamente. La respuesta del haz de electrones a la fuerza magnética periódica es seguir una trayectoria ondulada con el mismo período del arreglo de magnetos.

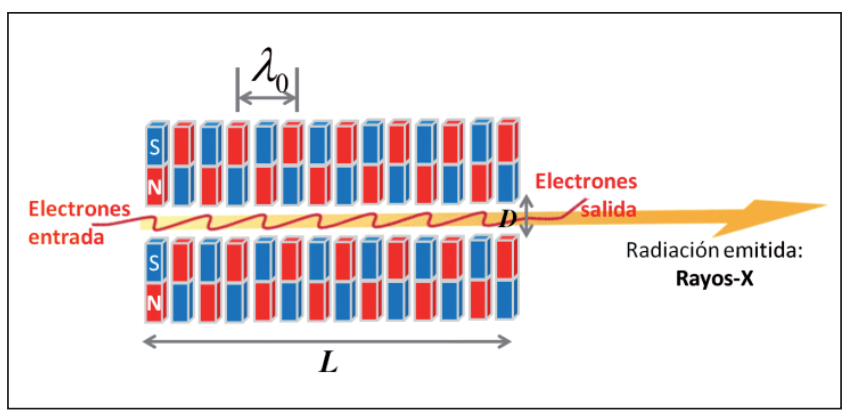

Figura 14: Ondulador de longitud $L$, período $\lambda_{0}$ y ancho de canal $D$. El campo magnético va alternando en polaridad, direcciones opuestas en arreglo periódico.
En la ondulación del haz de electrones, en las porciones alrededor de los máximos de la ondulación se tiene trayectoria curvada con aceleración centrípeta causante de radiación de sincrotrón, tangencial a la ondulación.

Con un arreglo de 50 a 100 magnetos, se superponen los conos de emisión de radiación de sincrotrón proveniente de cada ondulación, dando como resultado un solo haz de radiación, cuyas propiedades pueden ser ajustadas mediante la configuración de magnetos y la energía del rayo de electrones que atraviesa la instalación. En el arreglo de magnetos se puede variar la distancia entre magnetos, esto es variar $\lambda_{0}$. También se puede variar el ancho $D$ del canal, al reducirlo aumenta la intensidad del campo magnético y con ello se curva más fuertemente el haz de electrones en cada ondulación, esto es el radio de curvatura se reduce, aumentando así la potencia radiada en cada ondulación $\left(P_{\text {rad }} \propto E^{4} / R^{2}\right)$.

Al final de la unidad de magnetos se ubica un electroimán que desvía a un lado el rayo de electrones, dejando entonces el haz de radiación resultante a lo largo del eje de la unidad como un rayo de radiación bien definido.

La ondulación el haz de electrones ocurre en un plano, horizontal en el ejemplo aquí presentado, así que la radiación resultante del ondulador está polarizada linealmente.

Se distingue entre onduladores y wigglers. En el caso de wigglers el haz de electrones oscila con desviación angular grande comparada con el ángulo de apertura del cono de radiación de sincrotrón $\theta=1 / \gamma$, así que se obtiene un abanico de radiación.

En los onduladores la desviación angular en cada ondulación es pequeña, así que los conos de radiación de sincrotrón de cada ondulación se superponen, dando el resultado de un solo cono de radiación muy colimado en dirección frontal, hacia adelante, esto es de ángulo de apertura del cono muy pequeño.

Para obtener la frecuencia de la radiación emitida por el ondulador valen las siguientes consideraciones: El período del ondulador $\lambda_{0}$ se mide en el sistema de laboratorio, pero en el marco de referencia de los electrones que inciden con $\gamma=E /\left(m_{0} c^{2}\right)$, el período del arreglo de magnetos aparece como $\lambda_{0} / \gamma$ por el efecto relativista de contracción de Lorentz, esto es un período notoriamente reducido. Así los electrones oscilan con frecuencia

$$
f_{\text {emitida }}=\frac{1}{T}=\frac{c}{\left(\lambda_{0} / \gamma\right)}=\frac{\gamma c}{\lambda_{0}} .
$$

Esta es la frecuencia emitida en el marco de referencia de los electrones, pero en el marco de laboratorio se observa una frecuencia mayor por efecto Doppler relativista de la fuente en movimiento acercándose al observador. El corrimiento Doppler relativista es: 


$$
\sqrt{\frac{1+v / c}{1-v / c}}=\frac{1+v / c}{\sqrt{1-v^{2} / c^{2}}}=\gamma \cdot(1+v / c) \approx 2 \gamma,
$$

asumiendo para electrones relativistas $v \approx c$.

Así la frecuencia de la radiación del ondulador observada en el marco de laboratorio es:

$$
f_{\text {Lab }}=f_{\text {emitida }} \cdot 2 \gamma=\frac{2 \gamma^{2} c}{\lambda_{0}}
$$

La frecuencia de la radiación del ondulador observada en el laboratorio tiene una dependencia cuadrática de la energía del haz de electrones que atraviesan el ondulador, va como $\gamma^{2}$, y además depende del período del arreglo de magnetos, va como $1 / \lambda^{0}$. Para una energía constante del haz de electrones $\gamma=$ const y para un arreglo de magnetos fijo, la frecuencia observada del ondulador resulta ser aproximadamente mono-cromática, esto es de frecuencia $f_{\text {lab }}$ con una pequeña contribución de sus armónicos, múltiplos enteros de $f_{\text {lab. }}$. De acuerdo con las necesidades del usuario se puede escoger esta frecuencia, ajustando la energía del haz de electrones en el anillo del sincrotrón.

La frecuencia de la radiación de ondulador puede llegar hasta valores muy grandes según sea la energía del haz de electrones y el período del ondulador. Para electrones de $1 \mathrm{GeV}$ y un ondulador de 5 metros de longitud con 100 magnetos, $\gamma-1957, \lambda_{0}=5 \mathrm{~cm}$, así que $f_{\text {lab }}=4.6 \cdot 10^{16} \mathrm{~Hz}$.

Característica de especial importancia que presentan onduladores y wigglers es su alta brillantez, que resulta de combinar tres factores: El flujo $F$ de fotones emitidos en el intervalo de tiempo $\Delta t$ por radiación de sincrotrón por los electrones en cada ondulación, el área de emisión $\sigma$, la divergencia angular $\Delta \theta$. Se da la brillantez [Wi05] para la fracción del $0.1 \%$ del "ancho de banda (AB)", esto es el $01 . \%$ del intervalo disponible de energías de los fotones y finalmente se normaliza a una corriente $i_{0}=1$ Amperio de electrones en el sincrotrón operando en modo de anillo de almacenamiento. Así que la brillantez $B$ se expresa como

$$
B=\frac{\text { fotones }}{\Delta t \cdot \sigma \cdot \Delta \theta \cdot 0.1 \% A B \cdot i_{0}}
$$

que con las unidades habituales se expresa como

$$
B=\frac{\text { fotones }}{s \cdot \mathrm{mm}^{2} \cdot \mathrm{mrad}^{2} \cdot 0.1 \% A B \cdot A} .
$$

Se logra aumentar la brillantez con un mayor número de magnetos en el ondulador, que implica mayor número de ondulaciones, cada una fuente de fotones, y con un mayor número de electrones en el bunch de electrones que atraviesa el ondulador.

\section{Ventajas de la Radiación de Onduladores}

Luego de 100 años del descubrimiento de los rayos-X por Wilhelm Konrad Röntgen la radiación de onduladores y wigglers viene a enriquecer el mundo de los rayos-X, con verdaderos "rayos". Con los tubos de descarga tipo Röntgen los rayos-X se emiten en todas las direcciones, lo que obliga a blindajes y colimadores para eliminar, o al menos para reducir la radiación en las direcciones no deseadas y poder definir un rayo. Además solo se aprovecha una pequeña fracción de la radiación emitida, fracción que para algunas aplicaciones resulta insuficiente. La radiación que se obtiene de los tubos Röntgen no es coherente y no presenta polarización.

De los tubos Röntgen se obtiene el continuo de energías de Bremsstrahlung a las que se superponen las energías discretas de las líneas características del material del ánodo del tubo [Br09]. El continuo de energías dificulta el análisis de radiografías y reduce su calidad, por efectos de "endurecimiento del haz" ("beam hardening"), que resulta de la dependencia de la energía que presenta el coeficiente de absorción de rayos-X por el material estudiado [Bu13]. Esto dificulta la determinación de espesores de muestras radiografiadas, a pesar de mediciones complementarias de corrección del efecto. En especial para tomografías [Ro12], para la reconstrucción tridimensional del interior de la muestra, la calidad del resultado se ve afectada por efectos de los límites alcanzables en la corrección de endurecimiento del haz.

Comparando los onduladores con los antiguos tubos de Röntgen, las ventajas que ofrece esta moderna fuente de rayos- $\mathrm{X}$, radiación de sincrotrón y onduladores, son significativas.

Se destaca la muy alta brillantez, porque el haz que se obtiene es la superposición coherente de radiación de múltiples fuentes, de cada una de las ondulaciones, emitida desde un área muy pequeña, que aproxima a fuente puntual, emitida con divergencia muy pequeña de solo décimas a centésimas de mili radianes, en intervalos de tiempo muy cortos dados por el rápido cruce de los bunches del sincrotrón a través del ondulador.

La radiación de onduladores resulta coherente, con todas las ventajas que esto implica, las mismas que hacen del rayo laser mejor fuente de luz que lámparas convencionales incoherentes. La radiación preveniente de onduladores constituye la primera fuente coherente de rayos-X.

La radiación del ondulador resulta aproximadamente monocromática, una frecuencia fundamental con pequeñas contribuciones de sus armónicos, con la ventaja de ser ajustable según las necesidades del usuario, ajustando la energía del haz del sincrotrón, esto es ajunstando $\gamma$, y según sea el período $\lambda_{0}$ del ondulador. 
Si el ondulador es un wiggler, que lleva a mayores ondulaciones del haz de electrones se obtiene una banda ancha de frecuencias en la radiación resultante, así que es posible satisfacer las diversas necesidades de diversos usuarios, según el ondulador o wiggler utilizado, y en un mismo anillo sincrotrón-acumulador de tercera generación con varios tramos rectos es posible ofrecer estas diversas opciones con diversos onduladores o wigglers.

La radiación emitida por el ondulador resulta polarizada linealmente, debido a que las ondulaciones del haz de electrones se mantienen siempre en un plano.

El rayo resultante del ondulador presenta una estructura de tiempo bien definida y constante, un rayo pulsado de alta frecuencia, pulsos cortos, resultado del paso de los bunches de electrones con rapidez cercana a la de la luz a través del ondulador. Esta estructura de tiempo es un gran atractivo para experimentos que aprovechan la dependencia temporal del haz.

Estabilidad es otra propiedad importante del haz de radiación, rayos-X, proveniente del ondulador: Operando el anillo sincrotrón como anillo de almacenamiento, esto es manteniendo constante el campo magnético del sincrotrón, el haz de electrones en la máquina presenta estabilidad por períodos prolongados de tiempo de varias horas, del orden de 10 horas continuas, con las mismas condiciones.

\section{Aplicaciones}

El sincrotrón de tercera generación operando en el modo de anillo de almacenamiento de electrones, en estado continuo, con onduladores insertados en los trayectos rectilíneos constituye hoy la fuente moderna de rayos- $\mathrm{X}$, que se convierte en una muy poderosa herramienta para la investigación y la innovación tecnológica en múltiples frentes, en

- física atómica, física molecular,

- física de materia condensada, [Bu03], [So98]

- ciencias de materiales, [Co94], [Po97], [Sp01]

- estudios de superficies,

- nanotecnología,

- electrónica, [Be86], [Hi03], [Ko03], [Pi00], [To98]

- físicoquímica, química, [Li10]

- microscopía, biofísica, biología, medicina,

- física médica, radiología, [Am05], [Su03]

- ciencias marinas,

- arqueología y ciencias forenses, paleontología, [Ta06]

- geología, geofísica, ciencias de la tierra,

- instrumentación y métodos experimentales [Br08], [Gr83], [He84], [Kr06], [Ro89]

- desarrollos industriales [Ha08], [Ha12].
Mirando hacia Colombia y contemplando la idea de lograr una instalación de sincrotrón de tercera generación operando en el país, se encuentra un panorama muy prometedor: En todas las áreas de investigación y desarrollo arriba mencionadas se trabaja en Colombia y todas podrían beneficiarse con esta herramienta moderna que ayuda para ser más competitivos a nivel mundial.

Así por ejemplo la investigación en física de materia condensada, en particular física de estado sólido, es de larga tradición en Colombia, esto es desde los comienzos de la física en Colombia hace ya 50 años. En la actualidad en la mayoría de las instituciones universitarias colombianas que cuentan con programas académicos de física hay grupos de investigación activos en esta área o en áreas afines, en ciencias de materiales. Interesantes ejemplos de aplicación en Colombia se tendrían en las aplicaciones de rayos-X para el estudio de materiales, desde difracción, fluorescencia, radiografías en 2D hasta tomografías axiales computarizadas resultando en imágenes 3D. Así a manera de ejemplos se tiene:

El análisis de la difracción de rayos-X por rocas provenientes de regiones esmeraldíferas en Colombia, para determinar la presencia de berilio y así también la presencia de esmeraldas en las muestras rocosas [Be10].

El estudio de la estructura de carbones activados, obtenidos de carbón bituminoso del norte de Colombia y tratados mediante procedimientos físicos y químicos. Los rayos-X permiten caracterizar la estructura resultante, la fracción cristalina, apilamiento de capas de grafeno, entre otros [Ga08].

La caracterización de arcillas colombianas: Estudios cuantitativos detallados, en la identificación y cuantificación de los minerales de arcillas presentes en las muestras, además del estudio de las estructuras que presentan [Mo05].

El control de la calidad del hormigón común que resulta de la mezcla de cemento, agua y áridos: La caracterización de la mezcla ya solidificada, endurecida, es realizable mediante los rayos-X [Qu14].

En el desarrollo de nuevas tecnologías para fuentes de energía nuevas y renovables (FENR) están los paneles solares, las celdas solares para aprovechamiento de la energía solar empleando sistemas fotovoltaicos [Ro82], [Ro08]. Allí también los rayos-X encuentran aplicación en el estudio de nuevos compuestos fotovoltaicos, en su caracterización, en el estudio de sus propiedades ópticas, estructurales y morfológicas [Ar12], [Bo13].

El desarrollo de la industria electrónica puede beneficiarse igualmente de los rayos- $\mathrm{X}$ provenientes de los sincrotrones de tercera generación. La inspección de los microcircuitos electrónicos mediante rayos-X de alta resolución constituye una necesidad, no solo para el producto final, los 
microcircuitos ya producidos, sino en su optimización desde el diseño, al tener en cuenta los resultados del control con rayos-X [Sh02].

También en la ingeniería biomédica: Tomografías de alta resolución para el desarrollo de materiales adecuados para injertos en el cuerpo humano, como por ejemplo injertos vasculares artificiales [Br06], [Br07].

\section{Posibilidades de instalación para Colombia}

El desarrollo tecnológico mundial en el área de aceleradores de partículas ha llevado a instalaciones cada vez más compactas, entre otros a aceleradores como sincrotrones y anillos acumuladores "de tercera generación", cuyos costos se van reduciendo. Se suma a esto el desarrollo de Colombia como país ya emergente, con crecimiento sostenido de su economía por largos años, en busca de creatividad e innovación tecnológica para ser competitivos internacionalmente. Se reduce así la brecha de décadas pasadas entre la tecnología de avanzada y las posibilidades de Colombia de llegar a ella.

En la Colombia de hoy es razonable pensar en tener localmente recursos de alta tecnología como aceleradores de partículas, que faciliten lograr la innovación anhelada. Hoy cabe pensar en crear en un centro de acelerador sincrotrón de partículas así:

- Centro de Radiación de Sincrotrón a partir de un acelerador sincrotrón de tercera generación para electrones hasta $3 \mathrm{GeV}$.

Este centro tendría principalmente carácter de servicio, tanto para las universidades, como para institutos de investigación, y en especial para la industria.

Comparativamente a la misma energía, la potencia de radiación de sincrotrón es en un factor $10^{13}$ mayor para electrones, que para protones. De ahí que la fuente de radiación de sincrotrón sea el sincrotrón de electrones, operando con campo magnético constante como anillo de almacenamiento de electrones a energía de hasta $2 \mathrm{GeV}$. La Figura 15 muestra un esquema de la instalación propuesta.

Se trata de un sincrotrón de forma hexagonal, que tiene en cada uno de sus vértices un electroimán dipolar que curva el haz de electrones en 60 grados. El radio de curvatura de cada uno de estos seis electroimanes es de $10 \mathrm{~m}$, lo que da una distancia entre vértices opuestos del hexágono de $20 \mathrm{~m}$ y una distancia entre aristas opuestas (paralelas) de $17 \mathrm{~m}$. Los electroimanes dipolares se limitan a desviar el haz en 60 grados y no tienen funciones de enfoque. En los extremos de cada electroimán dipolar se ubica una lente magnética para enfoque del haz, cada lente compuesta por dos cuadrupolos, rotados 90 grados entre ellos, para enfoque en las dos direcciones transversales al haz (direcciones $X, Z$ ). Se tiene así una configuración de 12 lentes magnéticas, para un total de
24 electroimanes cuadrupolares. Entre vértices los trayectos del haz son rectilíneos de $10 \mathrm{~m}$ de longitud, las aristas del hexágono, que dan espacio para instalar en cuatro de ellas un ondulador de $3 \mathrm{~m}$ de longitud, cada uno centrado en la arista. Las otras dos aristas del hexágono quedan ocupadas, una con la entrada del haz del pre acelerador Linac, otra con la cavidad de radiofrecuencia RF, que acelera las partículas.

De cada uno de los cuatro onduladores propuestos se extrae un haz de fotones, rayos-X duros, aproximadamente monocromáticos, cada uno en un cono muy colimado. Cada ondulador puede ser configurado en sus parámetros $\left(\lambda_{0}, D\right.$, $L)$ de modo diferente a los otros, así que se tiene para cada estación de experimentos condiciones de rayo diferentes, acordes con las necesidades de los usuarios.

El pre acelerador es un acelerador lineal Linac de electrones de $8 \mathrm{~m}$ de longitud que alcanza una energía de $200 \mathrm{MeV}$, que es la energía inicial con la que ingresan los electrones al sincrotrón. Un electroimán bipolar de 90 grados desvía el haz del acelerador lineal Linac para la inyección al sincrotrón.

El anillo hexagonal opera inicialmente como acelerador sincrotrón aumentando la energía de los electrones en un factor de 10 , llevándolos de $0.2 \mathrm{GeV}$ hasta $2 \mathrm{GeV}$, lo que se logra aumentando el campo magnético de los dipolos. También los electroimanes cuadrupolares de las lentes magnéticas van siendo ajustados mientras va en aumento la energía de los electrones. Alcanzada la energía final, el campo magnético del acelerador se mantiene constante y la máquina opera como anillo de almacenamiento, manteniendo todos los parámetros

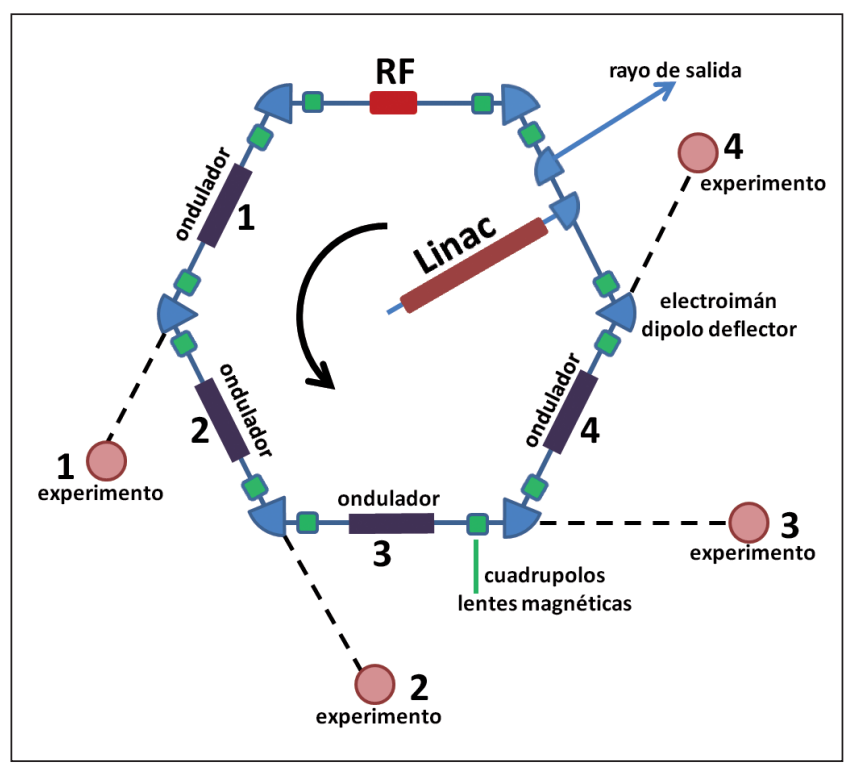

Figura 15: Instalación de fuente radiación de sincrotrón propuesta: Sincrotrón de electrones de tercera generación para electrones de energía máxima de $2 \mathrm{GeV}$. Configuración en hexágono. 
constantes. La cavidad de radiofrecuencia RF permanece siempre activa, también en el modo de estado constante de anillo de almacenamiento para compensar por la energía que los electrones van perdiendo por radiación de sincrotrón.

Finalizada la operación del anillo de almacenamiento, luego de varias horas de operación, se extrae el haz de la máquina mediante un electroimán de 90 grados ubicado también en la arista de inyección. Para electrones es realizable mantener el haz circulando en el anillo por intervalos de tiempo de varias horas, aproximadamente 5 horas, luego de las cuales es necesario renovar el haz.

El haz de salida de electrones del sincrotrón puede ser utilizado también como haz principal externo y llevado a un área experimental dedicada al haz de electrones de energía hasta $2 \mathrm{GeV}$, por ejemplo para experimentos dispersión de electrones.

\section{Infraestructura requerida}

Instalaciones de aceleradores de partículas solo son posibles si se cuenta con una infraestructura sólida, adecuada a las exigencias, sobre la cual se pueda construir y mantener funcionando el centro propuesto. Debe tenerse en cuenta, que no se trata solo del acelerador con sus equipos de soporte para su funcionamiento, sino también de su entorno en equipos e infraestructura en los experimentos que se realicen con el acelerador. Están también el personal, sus actividades y los recursos financieros.

Detallando la infraestructura de soporte para el acelerador está:

La tecnología de vacío, porque el movimiento de las partículas solo se lleva a cabo en condiciones de alto vacío. Está el alto vacío a lo largo del anillo del sincrotrón, que en la configuración de hexágono propuesta cada arista del hexágono se evacúa independientemente, para mantener una presión de $10^{-8}$ Torr. Esta es una tecnología perfectamente realizable en Colombia, donde existe ya la experiencia, personal conocedor, para quienes los equipos de vacío son de fácil adquisición, instalación y mantenimiento.

La criogenia, la infraestructura de bajas temperaturas requeridas al nivel de temperaturas del nitrógeno líquido, 77.4 Kelvin. El helio líquido para temperaturas de 4.2 Kelvin no es una exigencia indispensable porque los electroimanes requeridos y la cavidad de radiofrecuencia son convencionales, no superconductores. Hoy se cuenta en Colombia con buena experiencia en bajas temperaturas, incluyendo instalaciones de criogenia para producción de nitrógeno líquido operando en muy buenas condiciones, así que este es un requisito que se cumple plenamente. ${ }^{8}$

\footnotetext{
${ }^{8}$ Universidad de los Andes, Departamento de Física, Unidad de Criogenia, Nitrógeno Líquido. http://fisica.uniandes.edu.co
}

Electrotecnia de potencia para las instalaciones eléctricas de potencia que se requieren, adecuadas para el suministro eléctrico para electroimanes por ejemplo y para el preacelerador Linac que inyecta electrones al sincrotrón. En este aspecto en Colombia se tiene el conocimiento, recursos y experiencia adecuados.

Electrotecnia de radiofrecuencia para trasmisores de frecuencias hasta cientos de Mega Hertz, para la cavidad de radiofrecuencia, técnica bien conocida y en permanente y muy exitosa aplicación en todos los trasmisores de radio y televisión que operan en Colombia.

Electrónica, microelectrónica, computación, comunicaciones, áreas que son de perfecto dominio en el mundo moderno actual, existentes ya en Colombia.

La seguridad radiológica es parte muy importante de la infraestructura requerida. En este aspecto hay ya larga trayectoria en Colombia con las instalaciones de medicina nuclear en funcionamiento en el país, con el sistema de monitoreo y control establecido en Colombia, a cargo del Servicio Geológico Colombiano ${ }^{9}$, división de seguridad nuclear y protección radiológica.

La infraestructura de soporte para los experimentos en la periferia del acelerador coincide con la requerida para el acelerador mismo. Pero es claro que el centro propuesto es un centro de servicio y que son los usuarios los responsables por los experimentos que allí realicen.

En experimentos que utilizan la radiación de sincrotrón se presenta el área de detectores de partículas y radiaciones ionizantes, que ya está en Colombia en desarrollo en diversas universidades, en los grupos de investigación y desarrollo en física nuclear y de altas energías, y en los grupos de electrónica y microelectrónica. Son áreas donde ya hay interesante y notable trabajo realizado, pero donde falta el campo de acción donde utilizar estos desarrollos, como por ejemplo un centro de acelerador de radiación de sincrotrón.

\section{Conclusiones}

Con los nuevos desarrollos en aceleradores de partículas de tercera generación, se acercan las posibilidades de Colombia para tener las herramientas avanzadas, requeridas para ser competitivos en el mundo científico tecnológico globalizado de hoy.

Con el centro de acelerador sincrotrón se concreta de forma directa la transferencia de tecnología de los grandes laboratorios de aceleradores, donde por más de veinticinco años físicos e ingenieros colombianos han estado activos participando en

\footnotetext{
$\overline{9}$ Servicio Geológico Colombiano - Seguridad Nuclear y Protección Radiológica. http://www.sgc.gov.co/
} 
los experimentos de frontera en la investigación básica sobre la estructura fundamental de la materia y sobre el origen y evolución del Universo [Av10].

La propuesta de un centro de radiación de sincrotrón en Colombia resulta razonable en la actualidad. De realizarse

- sería un paso importante para lograr condiciones que permitan ser competitivo internacionalmente,

- sería un escenario para investigación y desarrollo, un punto de encuentro universidad-industria,

- sería motivo para que empresas internacionales realicen inversiones en nuestro país,

- sería contribución como generador de empleo, creando oportunidades para jóvenes altamente capacitados, para jóvenes científicos, investigadores y técnicos, quienes tendrían dónde realizar su actividad en nuestro país, para ser protagonistas del avance en ciencia, tecnología e innovación, reduciéndose así la fuga de cerebros.

La sola idea de esta "empresa", es ya un motivador para impulsar la actividad en ciencia y tecnología, para unir esfuerzos, para acercar diversas áreas de la ciencia y la industria en torno a un gran proyecto.

\section{Agradecimientos}

El autor agradece al Grupo de Física de Altas Energías de la Universidad de los Andes y al continuo apoyo del Departamento de Física y de la Facultad de Ciencias de la Universidad de los Andes. Agradecimientos también a COLCIENCIAS por el apoyo de largos años. Especiales agradecimientos a los laboratorios Fermilab (Batavia, Illinois, Estados Unidos) y CERN (Ginebra, Suiza), porque es con las actividades conjuntas, en el marco de las colaboraciones internacionales, que surge la motivación para el tema aquí presentado.

\section{Referencias}

\section{Orígenes y evolución de aceleradores: Artículos históricos}

[Bo46]D. Bohm, L. Foldy, "The Theory of the Synchrotron", Physical Review, Vol. 70, No. 5 and 6, September 1 and 15, 1946, p. $249-258$.

[Co32] J. D. Cockcroft, E. T. S. Walton, "Experiments with High Velocity Positive Ions - Further developments in the method of obtaining High Velocity Positive Ions", Communicated by Lord Rutherford, Proceedings of the Royal Society, A, Vol. 136, February 23, 1932, p. $619-630$.

[Co52] Ernest D. Courant, Stanley Livingston, Hartland S. Snyder, "The Strong-Focusing Synchrotron - A New High Energy Accelerator", Physical Review, Vol.88, No.5, December 1, 1952, p. $1190-1196$.

[Co57] E. D. Courant and H. S. Snyder, "Theory of the AlternatingGradient Synchrotron”, (July 15, 1957), Annals of Physics, Vol. 281, 2000, p. $360-408$.
[Co58]Ernest D. Courant, Hartland S. Snyder, "Theory of the Alternating-Gradient Synchrotron", Annals of Physics, No.3, 1958, p. $1-48$.

[Gr33]R. J. Van de Graaff, K. T. Compton, L. C. Van Atta, "The Electrostatic Production of High Voltage for Nuclear Investigations", Physical Review, Vol. 43, No. 3, February 1, 1933, p. $149-157$.

[Ke40] D. W. Kerst, "Acceleration of Electrons by Magnetic Induction”, Physical Review, Vol. 58, November 1, 1940, p. 841 .

[Ke41] D. W. Kerst, "The Acceleration of Electrons by Magnetic Induction", Physical Review, Vol. 60, July 1, 1941, p. 47 53.

[Ke42] D. W. Kerst, "Electronic Orbits in the Induction Accelerator", Physical Review, Vol. 60, July 1, 1941, p. 53 - 58.

[La32]Ernest O. Lawrence, Stanley Livingston, "The Production of High Speed Light Ions without the Use of High Voltages", Physical Review, Vol. 40, April 1, 1932, p. 19 - 35.

[La36] Ernest O. Lawrence, Donald Cooksey, "On the Apparatus for the Multiple Acceleration of Light Ions to High Speeds", Physical Review, Vol. 50, December 15, 1936, p. 1131 1140.

[La51] Ernest O. Lawrence, "The evolution of the cyclotron", Nobel Lecture, December 11, 1951.

[Mc45] Edwin M. McMillan, "The Synchrotron - A Proposed High Energy Particle Accelerator", University of California, Berkeley, California, Physical Review, September 5, 1945, p.143-144.

[Mc46] Edwin M. McMillan, "Radiation from a Group of Electrons Moving in a Circular Orbit", University of California, Berkeley, California, Physical Review, Vol.68, September 5, 1945, p.144-145.

[S131] David H. Sloan, Ernest O. Lawrence, "The Production of Heavy High Speed Ions without the Use of High Voltages" - (Linear Accelerator), Physical Review, Vol. 38, December 1, 1931, p. $2012-2032$.

[Ve44] Vladimir I. Veksler, "A new method of accelerating relativistic particles", Comptes Rendus de l'Academie Sciences de l'URSS, Vol.43, No.8, 1944, p. 329 - 331.

[Ve56] Vladimir I. Veksler et al., "The 10 BeV Proton Synchrotron of the Academy of Sciences USSR", All-Union Conference on the Physics of High Energy Particles, Academy of Sciences USSR, Moscow, 1956.

[Wa94]Pedro Waloschek, "The Infancy of Particle Accelerators: Life and Work of Rolf Wideröe", Vieweg \& Sohn Verlagsgesellschaft mbH, Brauschweig, Wiesbaden, 1994, ISBN-3-528-06586-9. DESY-Report 94-039, DESY, Hamburg, 1994, ISSN-0418-9833.

[Wi23] Rolf Wideröe, "Wideröe's original copy-books from 1923 to 1928”, ETH-Libr., Zurich, Hs 903: p. 633 - 638. 
[Wi28]Rolf Wideröe, "Über ein neues Prinzip zur Herstellung hoher Spannungen", Archiv für Elektrotechnik, Vol. 21, 1928 , p.387.

Textos sobre aceleradores de partículas

[Co08] Mario Conte, William W. MacKay, "An Introduction to the Physics of Particle Accelerators", World Scientific Publishing Co., Singapore, 2008, ISBN-13-978-981-277-961-8.

[Ha12] Robert W. Hamm, Marianne E. Hamm, "Industrial Accelerators and their Applications", World Scientific Publishing Co., Singapore, 2012, ISBN-978-981-4307-04-8.

[Ja99] John David Jackson, "Classical Electrodynamics", Third Edition, John Wiley \& Sons, Inc., New York, 1999, ISBN0-471-30932-X.

[Li62] Stanley Livingston, John P. Blewett, "Particle Accelerators", McGraw-Hill Book Co., New York, 1962, Library of Congress Catalog Card Number 61-12960.

[Se07] Andrew Sessler, Edmund Wilson, "Engines of Discovery", World Scientific Publishing Co.,Singapore, 2007, ISBN-13978-981-270-071-1, ISBN-10-981-270-071-4.

[Wi05] Klaus Wille, "The Physics of Particle Accelerators - An Introduction", Oxford University Press, Oxford, 2005, ISBN-0-19-850549-3.

[Wi06] Edmund Wilson, "An Introduction to Particle Accelerators", Oxford University Press, Oxford, 2006, ISBN-0-19-850829-8.

\section{Aplicaciones de aceleradores y fuentes de radiación de} sincrotrón

\section{Instrumentación y métodos experimentales}

[Br08] Oliver Brunke ; Kathleen Brockdorf ; Susanne Drews ; Bert Müller ; Tilman Donath ; Julia Herzen ; Felix Beckmann, "Comparison between x-ray tube-based and synchrotron radiation-based $\mu$ CT", Proc. SPIE 7078, Developments in X-Ray Tomography VI, 70780 U (September 16, 2008); doi:10.1117/12.794789.

[Br09] Jeff C. Bryan, "Introduction to Nuclear Science", CRC Press, Taylor \& Francis Group, Boca Raton, Florida, 2009, ISBN-978-1-4200-6164-2.

[Bu13] C. Bula Villarreal, B. Gómez Moreno, "Beam Hardening Correction for Signal to Thickness Calibration in Digital Radiographies with Medipix2 Detector", Revista Colombiana de Física, Vol. 45, No.1 de 2013, pp. 54 - 59.

[Gr83] L. Grodzins, “Optimum energies for x-ray transmission tomography of small samples: Applications of synchrotron radiation to computerized tomography", Nuclear Instruments and Methods in Physics Research, Volume 206, Issue 3, 1 March 1983, Pages 541-545.

[He84] J R Helliwell, "Synchrotron X-radiation protein crystallography: instrumentation, methods and applications", Review Article, Reports on Progress in Physics, Volume 47, Number 11, (1984), p.1403. doi:10.1088/00344885/47/11/001.
[Kr06] M. Krumrey, M. Gerlach, F. Scholze, G. Ulm, "Calibration and characterization of semiconductor X-ray detectors with synchrotron radiation", Nuclear Instruments and Methods in Physics Research Section A: Accelerators, Spectrometers, Detectors and Associated Equipment, Volume 568, Issue 1, 30 November 2006, pp. 364-368.

[Ro12]C.F. Roa García, B. Gómez Moreno, “Tomography with Medipix2 Semiconductor Pixel Detector", Revista Colombiana de Física, Vol. 43, No.2 de 2011, pp. 318 - 322.

[Ro89] Brian Rodricks, Roy Clarke, Robert Smither and Alain Fontaine, "A virtual phase CCD detector for synchrotron radiation applications", Review of Scientific Instruments > Volume 60, Issue 8, 2586 (1989); http://dx.doi.org/10. 1063/1.1140675.

\section{Materia condensada y aplicaciones diversas}

[Bu03] T Buonassisi, M Heuer, O.F Vyvenko, A.A Istratov, E.R Weber, Z Cai, B Lai, T.F Ciszek, R Schindler, “Applications of synchrotron radiation X-ray techniques on the analysis of the behavior of transition metals in solar cells and singlecrystalline silicon with extended defects", Physica B: Condensed Matter, Volumes 340-342, 31 December 2003, pp. 1137-1141.

[So98] Y. L. Soo, S. Huang, Y. H. Kao and A. D. Compaan, "Investigation of interface morphology and composition mixing in $\mathrm{CdTe} / \mathrm{CdS}$ heterojunction photovoltaic materials using synchrotron radiation", Journal of Applied Physics, Volume 83, Issue 8, 1998, pp.4173, http://dx.doi.org/10. 1063/1.367171.

\section{Electrónica, microelectrónica e industria de semiconductores}

[Be86] E.W. Becker, W. Ehrfeld, P. Hagmann, A. Maner, D. Münchmeyer, "Fabrication of microstructures with high aspect ratios and great structural heights by synchrotron radiation lithography, galvanoforming, and plastic moulding (LIGA process)", Microelectronic Engineering, Volume 4, Issue 1, May 1986, Pages 35-56.

[Hi03] Yoshihiro Hirata, "LIGA process - micromachining technique using synchrotron radiation lithography - and some industrial applications", Nuclear Instruments and Methods in Physics Research Section B: Beam Interactions with Materials and Atoms, Ionizing Radiation and Polymers, Volume 208, August 2003, Pages 21-26.

[Ko03]K. Kobayashi, M. Yabashi, Y. Takata, T. Tokushima, S. Shin, K. Tamasaku, D. Miwa, T. Ishikawa, H. Nohira, T. Hattori, Y. Sugita, O. Nakatsuka, A. Sakai and S. Zaima, "High resolution-high energy $\mathrm{x}$-ray photoelectron spectroscopy using third-generation synchrotron radiation source, and its application to Si-high k insulator systems", Appl. Phys. Lett. 83, 1005 (2003); http://dx.doi.org/10.1063/1.1595714.

[Pi00] P Pianetta, K Baura, A Singha, S Brennan, Jonathan Kerner, D Werho, J Wang, "Application of synchrotron radiation to TXRF analysis of metal contamination on silicon wafer surfaces", Proceedings of the 11th International Conference on Thin Films, Thin Solid Films, Volume 373, Issues 1-2, 3 September 2000, Pages 222-226. 
[To98] D W L Tolfree, "Microfabrication using synchrotron radiation", Reports on Progress in Physics Volume 61 Number 4, 1998, p.313.

\section{Ciencia de materiales y aplicaciones en la industria}

[Co94] David C. Copley M.A., Jeffrey W. Eberhard Ph.D., Gregory A. Mohr Ph.D., "Computed tomography part I: Introduction and industrial applications", JOM, The Journal of The Minerals, Metals \& Materials Society (TMS), January 1994, Volume 46, Issue 1, pp 14-26.

[Ha08] Robert W. Hamm., "Industrial Accelerators", Reviews of Accelerator Science and Technology, Vol. 1 (2008), pp 163 $-184$.

[Po97] H. F. Poulsen, S. Garbe, T. Lorentzen, D. Juul Jensen, F. W. Poulsen, N. H. Andersen, T. Frello, R. Feidenhans'l and H. Graafsma, "Applications of High-Energy Synchrotron Radiation for Structural Studies of Polycrystalline Materials", Journal of Synchrotron Radiation, Volume 4, Part 3 (May 1997), pp.147-154.

[Sp01] P. Spanne, J. F. Thovert, C. J. Jacquin, W. B. Lindquist, K. W. Jones, and P. M. Adler, "Synchrotron Computed Microtomography of Porous Media: Topology and Transports", Phys. Rev. Lett. 73, 2001.

\section{Química}

[Li10] Yuyang Li and Fei Qi, "Recent Applications of Synchrotron VUV Photoionization Mass Spectrometry: Insight into Combustion Chemistry", Accounts of Chemical Research 201043 (1), 68-78.

\section{Paleontología}

[Ta06] P. Tafforeau, R. Boistel, E. Boller, A. Bravin, M. Brunet, Y. Chaimanee, P. Cloetens, M. Feist, J. Hoszowska, J.J. Jaeger, R.F. Kay, V. Lazzari, L. Marivaux, A. Nel, C. Nemoz, X. Thibault, P. Vignaud, S. Zabler, "Applications of X-ray synchrotron microtomography for non-destructive 3D studies of paleontological specimens", Applied Physics A, May 2006, Volume 83, Issue 2, pp 195-202.

\section{Aplicaciones médicas}

[Am05] Ugo Amaldi and Gerhard Kraft, "Recent applications of Synchrotrons in cancer therapy with Carbon Ions", Europhysics News, Volume 36, Number 4, July-August 2005.

[Su03] P Suortti and W Thomlinson, "Medical applications of synchrotron radiation", Physics in Medicine and Biology, Volume 48, Number 13, Received 24 January 2003, Published 17 June 2003

\section{Posibles aplicaciones en Colombia}

[Ar12] C.A. Arredondo, W. Vallejo, J. Hernandez, G. Gordillo, "In $(\mathrm{O}, \mathrm{OH}) \mathrm{S} / \mathrm{AgInS} 2$ absorbent layer/buffer layer system for thin film solar cells", Photovoltaic Specialists Conference (PVSC), 38th IEEE, 3-8 June 2012.
[Be10] D. Betancourth G., J. F. Gómez C., J. C. Mosquera, L. Tirado-Mejía, "Análisis por difracción de rayos x de rocas provenientes de región esmeraldífera”, Scientia et Technica, ISSN 0122-1701, Vol. 1, No. 44, 2010, pp. 257-260. Trabajo conjunto de la Universidad Tecnológica de Pereira y la Universidad del Quindío, Colombia.

[Bo13] M. A. Botero-Londoño, G. Gordillo, C. Calderon, "Preparación y estudio de películas delgadas de $\mathrm{ZnS}$ y ZnS:In", Revista Colombiana de Física, Vol 45, No 2 (2013), pp.168 - 171 .

[Br06] J.C.Briceño-Triana, A.E.García-Torres, O. Solano, D.M. Sánchez, D.Piñeros, R.Beltrán, "Use of porcine small intestine submucosa conduits as a vascular graft: Initial in vivo results", ed. Lippincott Williams \& Wilkins, Asaio Journal ISSN: 1058-2916, vol.52, fasc.2, 2006, pp.13A-13A.

[Br07] J.C. Briceño-Triana, J.A. Arias, A. Zuluaga, M. Hernández, "Hemodynamics of Carotid Stenosis: A Diagnostic Image Characterization and Computational Study", ed. Lippincott Williams \& Wilkins, Asaio Journal ISSN: 1058-2916, vol.52, fasc.2, 2006, pp.57A - 57A.

[Ga08] A. A. García, P. Y. Moreno, J. A. García, L. Giraldo, J.C. Moreno-Piraján, "Estudio de parámetros estructurales en la carbonización y activación de un carbón colombiano mediante difraccion de rayos X", Revista de química teórica y aplicada, ISSN 0001-9704, Vol. 65, No. 535, 2008, pp. 230-235.

[Mo05] J. Mojica, J.J. Ipus, G.A. Pérez-Alcázar, “Caracterización de arcillas colombianas por espectroscopía Mössbauer y difracción de rayos-X", Revista de la Sociedad Colombiana de Física, ISSN-e 0120-2650, Vol. 37, №. 1, 2005, pp. 187-190.

[Qu14]H. P. Quiroz, A. Dussan, D.A. Landínez Téllez, J. RoaRojas, Universidad Nacional de Colombia - Sede Bogotá, "Caracterización del hormigón de 3000psi (20 mpa) utilizando difracción de rayos X", Cemento Hormigón, ISSN 0008-8919, Nº. 961, 2014 , pp. 52-55.

[Ro82] H. Rodríguez M., "Estado Actual de la I\&D de las FENR en Colombia”, Colciencias, 1982, pp. 263.

[Ro08]H. Rodríguez M., "Desarrollo de la energía solar en Colombia y sus perspectivas", Revista de ingeniería. Universidad de los Andes. Bogotá, Colombia., rev.ing. ISSN. 0121-4993. Nov. 2008.

[Sh02]F. Shlieper, “Análisis avanzado de componentes electrónicos y conexiones utilizando tecnología microfocus de rayos X", Revista Española de Electrónica, ISSN 0482-6396, № 568, 2002, pp. 58-62.

\section{Colombianos activos con Aceleradores de Partículas}

[Av10] C. Ávila Bernal, B. Gómez Moreno, "Grupo de Física de Altas Energías de la Universidad de los Andes: Veinte años de investigación en la frontera de las altas energías", Colección Sello 60 Años, Universidad de los Andes, Ediciones Uniandes, Abril de 2010, ISBN 978-958-695-477-8. 